\title{
SEQUENTIAL PRODUCTS OF QUANTUM MEASUREMENTS
}

\author{
Stan Gudder \\ Department of Mathematics \\ University of Denver \\ Denver, Colorado 80208 \\ sgudder@du.edu
}

\begin{abstract}
Our basic structure is a finite-dimensional complex Hilbert space $H$. We point out that the set of effects on $H$ form a convex effect algebra. Although the set of operators on $H$ also form a convex effect algebra, they have a more detailed structure. W introduce sequential products of effect and operations. Although these have already been studied, we introduce the new concept of sequential products of effects with operations and operations with effects. We then consider various special types of operations. After developing properties of these concepts, the results are generalized to include observables and instruments. In particular, sequential products of observables with instruments and instruments with observables are developed. Finally, we consider conditioning and coexistence of observables and instruments.
\end{abstract}

\section{Basic Definitions}

Let $H$ be a finite-dimensional complex Hilbert space.The basic concepts of the present version of quantum measurement theory are the sets of effects $\mathcal{E}(H)$, states $\mathcal{S}(H)$ and operations $\mathcal{O}(H)$ on $H$ [1, 8, 10, 13]. Denoting the set of linear operators on $H$ by $\mathcal{L}(H)$, we define $\mathcal{E}(H)=\{a \in \mathcal{L}(H): 0 \leq a \leq I\}$ where $0, I$ are the zero and identity operators, respectively. An effect $a \in$ $\mathcal{E}(H)$ corresponds to a two-valued yes - no experiment. The effect $a^{\prime}=I-a$ 
has the value yes if $a$ has the value no and we call $a^{\prime}$ the complement of $a$. If $a, b \in \mathcal{E}(H)$ and $a+b \in \mathcal{E}(H)$, then we write $a \perp b$. When $a \perp b$, we interpret $a+b \in \mathcal{E}(H)$ as the parallel sum of the effects $a$ and $b$. The effect 0 always has value no and $I$ always has value yes. The fact that $a \perp a^{\prime}$ and $a+a^{\prime}=I$ indicates that either $a$ or $a^{\prime}$ has the value yes but not both. An element $\rho \in \mathcal{E}(H)$ that satisfies $\operatorname{tr}(\rho)=1$ is called a state. States describe the initial condition of a quantum system. If $a \in \mathcal{E}(H)$ and $\rho \in \mathcal{S}(H)$, then the probability that $a$ has value yes when the system is in state $\rho$ is given by $\mathcal{P}_{\rho}(a)=\operatorname{tr}(\rho a)$. It is clear that $\mathcal{P}_{\rho}(0)=0, \mathcal{P}_{\rho}(I)=1$ and if $a \perp b$, then $\mathcal{P}_{\rho}(a+b)=\mathcal{P}_{\rho}(a)+\mathcal{P}_{\rho}(b)$.

A linear map $\mathcal{I}: \mathcal{L}(H) \rightarrow \mathcal{L}(H)$ is completely positive if $\mathcal{I} \otimes I: H \otimes K \rightarrow$ $H \otimes K$ is positive for every auxiliary finite dimensional Hilbert space $K$. We call $\mathcal{I}: \mathcal{L}(H) \rightarrow \mathcal{L}(H)$ an operation if $\mathcal{I}$ is completely positive and $\operatorname{tr}[\mathcal{I}(\rho)] \leq$ $\operatorname{tr}(\rho)$ for every positive $\rho \in \mathcal{L}(H)[1,8,10,13$. An operation $\mathcal{I}$ is called a channel if $\operatorname{tr}[\mathcal{I}(\rho)]=\operatorname{tr}(\rho)$ for every positive $\rho \in \mathcal{L}(H)$. In particular, if $\rho \in \mathcal{S}(H)$ then $\operatorname{tr}[\mathcal{I}(\rho)]=1$. Any $\mathcal{I} \in \mathcal{O}(H)$ has a Kraus decomposition $\mathcal{I}(\rho)=\sum_{i=1}^{n} A_{i} \rho A_{i}^{*}$ where $A_{i} \in \mathcal{L}(H), i=1,2, \ldots, n$ [1, 8, 10, 13]. The Kraus operators $A_{i}$ need not be unique. Since $\operatorname{tr}[\mathcal{I}(\rho)] \leq \operatorname{tr}(\rho)$ we have that

$$
\operatorname{tr}\left(\rho \sum A_{i}^{*} A_{i}\right)=\sum \operatorname{tr}\left(\rho A_{i}^{*} A_{i}\right)=\sum \operatorname{tr}\left(A_{i} \rho A_{i}^{*}\right)=\operatorname{tr}[\mathcal{I}(\rho)] \leq \operatorname{tr}(\rho I)
$$

for every positive $\rho$. It follows that $\sum A_{i}^{*} A_{i} \leq I$. An operation is thought of as an apparatus $\mathcal{I}$ that can be employed to measure an effect $\widehat{\mathcal{I}}$. We define the probability that $\mathcal{I}$ has value yes in the state $\rho$ to be $\mathcal{P}_{\rho}(\mathcal{I})=$ $\operatorname{tr}[\mathcal{I}(\rho)]$ and $\widehat{\mathcal{I}}$ is the unique effect satisfying $\operatorname{tr}(\rho \widehat{\mathcal{I}})=\mathcal{P}_{\rho}(\mathcal{I})$ for all $\rho \in$ $\mathcal{S}(H)$ and say that $\mathcal{I}$ measures $\widehat{\mathcal{I}}$. Although every $\mathcal{I} \in \mathcal{O}(H)$ measures a unique $\widehat{\mathcal{I}} \in \mathcal{E}(H)$, an effect is measured by many operations. That is, many apparatuses can be employed to measure an effect $a$. Moreover, $\mathcal{I}$ gives more information than $\widehat{\mathcal{I}}$ because $\mathcal{I}(\rho) / \operatorname{tr}[\mathcal{I}(\rho)]$ is the updated state after $\mathcal{I}$ (or $\widehat{\mathcal{I}}$ ) is measured (assuming $\operatorname{tr}[\mathcal{I}(\rho)] \neq 0$ ). One way to specify $\widehat{\mathcal{I}}$ is the following: If $\mathcal{I}$ has Kraus decomposition $\mathcal{I}(\rho)=\sum A_{i} \rho A_{i}^{*}$, then by (1.1) we have that $\widehat{\mathcal{I}}=\sum A_{i}^{*} A_{i}$. This also shows that if $\mathcal{I}(\rho)=\sum B_{j} \rho B_{j}^{*}$ is another Kraus decomposition for $\mathcal{I}$, then $\sum B_{j}^{*} B_{j}=\sum A_{i}^{*} A_{i}$.

We now consider the mathematical structures of $\mathcal{E}(H)$ and $\mathcal{O}(H)$. Let $(V, \leq)$ be a finite-dimensional ordered real linear space [8, 13] and let $u \in V$ 
satisfy $u>0$. Letting $E$ be the ordered interval

$$
E=[0, u]=\{x \in V: 0 \leq x \leq u\}
$$

we call $(E, 0, u)$ a convex effect algebra [7]. For $x, y \in E$ we write $x \perp y$ if $x+y \in E$. It is easy to check that $E$ satisfies the effect algebra axioms:

(1) If $x \perp y$, then $y \perp x$ and $x+y=y+x$.

(2) If $y \perp z$ and $x \perp(y+z)$, then $x \perp y, z \perp(x+y)$ and we have $x+(y+z)=(x+y)+z$.

(3) For every $x \in E$ there exists a unique $x^{\prime} \in E$ such that $x \perp x^{\prime}$ and $x+x^{\prime}=u$.

(4) If $x \perp u$, then $x=0$.

Moreover, $E$ is convex because if $x_{1}, \ldots, x_{n} \in E$ and $\lambda_{i} \geq 0$ with $\sum \lambda_{i}=1$, then $\sum \lambda_{i} x_{i} \in E$. Indeed, we have that

$$
0 \leq \sum \lambda_{i} x_{i} \leq \sum \lambda_{i} u=u
$$

We call the element $x^{\prime}$ in (3) the complement of $x$. If $E=[0, u]$ and $F=[0, v]$ are convex effect algebras, a map $J: E \rightarrow F$ is an isomorphism if $J$ is an order-preserving bijection, $J(u)=v, J(x+y)=J(x)+J(y)$ whenever $x \perp y$ and $J\left(\sum \lambda_{i} x_{i}\right)=\sum \lambda_{i} J\left(x_{i}\right)$ whenever $\lambda_{i} \geq 0, \sum \lambda_{i}=1$.

Notice that $\mathcal{E}(H)$ is a convex effect algebra because $\mathcal{E}(H)=[0, I] \subseteq$ $\mathcal{L}_{\mathbb{R}}(H)$ where $\mathcal{L}_{\mathbb{R}}(H)$ is the real linear span of $\mathcal{E}(H)$. In a similar way, letting $V$ be the real linear span of $\mathcal{O}(H)$ we have that $\mathcal{O}(H)=[0, I] \subseteq V$ where 0 and $I$ are the zero and identity operations, respectively, so $\mathcal{O}(H)$ is also a convex effect algebra. However, this convex effect algebra does not specify the detailed probabilistic structure of $\mathcal{O}(H)$. Although $I \in \mathcal{E}(H)$ is the unique certain effect, the identity $I \in \mathcal{O}(H)$ is not the unique certain operation. If $\mathcal{C}$ is a channel, then for any $\rho \in \mathcal{S}(H)$ we have that $\mathcal{P}_{\rho}(\mathcal{C})=$ $\operatorname{tr}[\mathcal{C}(\rho)]=1$ so $\mathcal{C}$ has value yes for any state so $\mathcal{C}$ is certainly yes. Similarly, if $\mathcal{I}, \mathcal{J} \in \mathcal{O}(H)$ and $\widehat{\mathcal{I}}=\widehat{\mathcal{J}}$ then

$$
\mathcal{P}_{\rho}(\mathcal{I})=\operatorname{tr}[\mathcal{I}(\rho)]=\operatorname{tr}(\rho \widehat{\mathcal{I}})=\operatorname{tr}(\rho \widehat{\mathcal{J}})=\operatorname{tr}[\mathcal{J}(\rho)]=\mathcal{P}_{\rho}(\mathcal{J})
$$

for every $\rho \in \mathcal{S}(H)$ so we cannot distinguish $\mathcal{I}$ and $\mathcal{J}$ probabilistically. For $\mathcal{I}, \mathcal{J} \in \mathcal{O}(H)$ we write $\mathcal{I} \approx \mathcal{J}$ if $\widehat{\mathcal{I}}=\widehat{\mathcal{J}}$. It is clear that $\approx$ is an equivalence 
relation in $\mathcal{O}(H)$. We denote the equivalence class containing $\mathcal{I}$ by $[\mathcal{I}]$ and the set of equivalence classes by $\mathcal{O}(H) / \approx$. We write $[\mathcal{I}]^{\wedge}=\widehat{\mathcal{I}}$ and it is clear that this is well-defined.

Theorem 1.1. $\mathcal{O}(H) / \approx$ is a convex effect algebra and $\wedge: \mathcal{O}(H) / \approx \rightarrow$ $\mathcal{E}(H)$ is an isomorphism.

Proof. We write $0=\{0\}=[0]$ and $u=[\mathcal{C}]$ where $\mathcal{C}$ is a channel and we have that $\widehat{0}=0$ and $\widehat{u}=I$. If $\mathcal{I}, \mathcal{J} \in \mathcal{O}(H)$ with $\mathcal{I} \perp \mathcal{J}$ we define $[\mathcal{I}]+[\mathcal{J}]=$ $[\mathcal{I}+\mathcal{J}]$ and if $\mathcal{I}_{i} \in \mathcal{O}(H), \lambda_{i} \geq 0$ with $\sum \lambda_{i}=1, i=1,2, \ldots, n$ we define

$$
\sum_{i} \lambda_{i}\left[\mathcal{I}_{i}\right]=\left[\sum \lambda_{i} \mathcal{I}_{i}\right]
$$

Moreover, we write $[\mathcal{I}] \leq[\mathcal{J}]$ if $\widehat{\mathcal{I}} \leq \widehat{\mathcal{J}}$. It is clear that these are well-defined. We then have that $0 \leq[\mathcal{I}] \leq u$ for all $\mathcal{I} \in \mathcal{O}(H)$. It is straightforward to show that $\mathcal{O}(H) / \approx=[0, u]$ is an order interval for a real ordered linear space $V$ consisting of the linear span of $\mathcal{O}(H) / \approx$ and hence is a convex effect algebra. Since $[\mathcal{I}+\mathcal{J}]^{\wedge}=\widehat{\mathcal{I}}+\widehat{\mathcal{J}}$ and

$$
\left(\sum \lambda_{i}\left[\mathcal{I}_{i}\right]\right)^{\wedge}=\left(\sum \lambda_{i} \mathcal{I}_{i}\right)^{\wedge}=\sum \lambda_{i} \widehat{\mathcal{I}}_{i}
$$

we have that $\wedge$ preserves + and convex combinations. Clearly, $\widehat{u}=I$ and $\wedge$ is order-preserving. If $a \in \mathcal{E}(H)$ we will show there exists an $\mathcal{I} \in \mathcal{O}(H)$ such that $\widehat{\mathcal{I}}=a$ so $\wedge$ is surjective. To show that $\wedge$ in injective, suppose that $[\mathcal{I}]^{\wedge}=[\mathcal{J}]^{\wedge}$. Then $\widehat{\mathcal{I}}=\widehat{\mathcal{J}}$ so $[\mathcal{I}]=[\mathcal{J}]$. Hence, ${ }^{\wedge}: \mathcal{O}(H) / \approx \rightarrow \mathcal{E}(H)$ is an isomorphism.

Besides sums and convex combinations, there is another important way of combining effects and operations. For $a, b \in \mathcal{E}(H)$ we define their sequential product $a \circ b=a^{1 / 2} b a^{1 / 2}$ where $a^{1 / 2}$ is the unique positive square-root of $a$ [3, 4]. It is easy to check that $a \circ b \leq a$ so we indeed have that $a \circ b \in \mathcal{E}(H)$. We interpret $a \circ b$ to be the effect resulting from first measuring $a$ and then measuring $b$. Then $\mathcal{E}(H)$ has the mathematical structure of a convex, sequential effect algebra [6, 7]. Because of the sequential order of $a \circ b$, the measurement of $a$ can influence (interfere) with the measurement of $b$ but not vice versa. This is emphasized by the fact that $a \circ(b+c)=a \circ b+a \circ c$ and $a \circ\left(\sum \lambda_{i} b_{i}\right)=\sum \lambda_{i} a \circ b_{i}$. We then say that $a \circ b$ is additive and affine in the second variable. In general, $a \circ b$ is not additive or affine in the first variable. 
Also, it can be shown that $a \circ b=b \circ a$ if and only if $a b=b a$ [4]. In a similar way, for $\mathcal{I}, \mathcal{J} \in \mathcal{O}(H)$ we define their sequential product $\mathcal{I} \circ \mathcal{J}(\rho)=\mathcal{J}(\mathcal{I}(\rho))$ [5, 7]. Although we still retain the same influence interpretation, $\mathcal{I} \circ \mathcal{J}$ is additive and affine in both variables. However, $\mathcal{I} \circ \mathcal{J} \neq \mathcal{J} \circ \mathcal{I}$, in general. We say that $a \in \mathcal{E}(H)$ is sharp if $a$ is a projection. It can be shown that $a$ is sharp if and only if $a \wedge a^{\prime}=0$. That is if $b \leq a, a^{\prime}$ then $b=0$. An effect $a$ is atomic if $a$ is a one-dimensional projection. The next result pertains to additivity and commutativity of sharp and atomic effects.

Theorem 1.2. (i) If $a_{1}, a_{2}, \ldots, a_{n}$ are sharp and $\sum a_{i}=I$, then $b=\sum a_{i} \circ b$ if and only if $b a_{i}=a_{i} b, i=1,2, \ldots, n$. (ii) If $a$ and $b$ are atomic and have the form $a=|\phi\rangle\langle\phi|, b=| \psi\rangle\langle\psi|$, then $\mathcal{P}_{\rho}(a \circ b)=\mathcal{P}_{\rho}(b \circ a)$ if and only if $\langle\phi, \rho \phi\rangle=\langle\psi, \rho \psi\rangle$ or $a b=0$.

Proof. (i) If $b a_{i}=a_{i} b, i=1,2, \ldots, n$, then

$$
\sum a_{i} \circ b=\sum a_{i} b a_{i}=\sum a_{i} b=b
$$

Conversely, suppose that $b=\sum a_{i} \circ b=\sum a_{i} b a_{i}$. Since $\sum a_{i}=I$, we obtain

$$
a_{j}+\sum_{i \neq j} a_{j} a_{i} a_{j}=a_{j}
$$

Hence, $\sum_{i \neq j} a_{j} a_{i} a_{j}=0$ so that $a_{j} a_{i} a_{j}=0, i \neq j$. Therefore,

$$
\left(a_{j} a_{i}\right)\left(a_{j} a_{i}\right)^{*}=a_{j} a_{i} a_{i} a_{j}=a_{j} a_{i} a_{j}=0
$$

It follows that $a_{j} a_{i}=0$ for $i \neq j$. But then

$$
a_{j} b=a_{j} b a_{j}=b a_{j}
$$

for $j=1,2, \ldots, n$. (ii) We have that $\mathcal{P}_{\rho}(a \circ b)=\mathcal{P}_{\rho}(b \circ a)$ if and only if

$$
\operatorname{tr}(\rho|\phi\rangle\langle\phi|| \psi\rangle\langle\psi|| \phi\rangle\langle\phi|)=\operatorname{tr}(\rho|\psi\rangle\langle\psi|| \phi\rangle\langle\phi|| \psi\rangle\langle\psi|)
$$

This is equivalent to

$$
\begin{aligned}
|\langle\phi, \psi\rangle|^{2}\langle\phi, \rho \phi\rangle & =|\langle\phi, \psi\rangle|^{2} \operatorname{tr}(\rho|\phi\rangle\langle\phi|)=|\langle\phi, \psi\rangle|^{2} \operatorname{tr}(\rho|\psi\rangle\langle\psi|) \\
& =|\langle\phi, \psi\rangle|^{2}\langle\psi, \rho \psi\rangle
\end{aligned}
$$

Since $\langle\phi, \psi\rangle=0$ if and only if $a b=0$, then the result follows.

It is easy to check that $a \in \mathcal{E}(H)$ is atomic if and only if $b \leq a$ implies that $b=\lambda a$ for some $\lambda \in[0,1]$. 


\section{Bayes' Rules}

First note that $\mathcal{P}_{\rho}$ has the usual properties of a probability measure on $\mathcal{E}(H)$. That is, $0 \leq \mathcal{P}_{\rho}(a) \leq 1$ for all $a \in \mathcal{E}(H), \mathcal{P}_{\rho}(I)=1$ and if $a \perp b$, then $\mathcal{P}_{\rho}(a+b)=\mathcal{P}_{\rho}(a)+\mathcal{P}(b)$. This last equation shows that $a \perp b$ is the analogue of disjointness of events in classical probability theory. For $a, b \in \mathcal{E}(H)$, it is natural to define the conditional probability

$$
\mathcal{P}_{\rho}(b \mid a)=\frac{\mathcal{P}_{\rho}(a \circ b)}{\mathcal{P}_{\rho}(a)}=\frac{\operatorname{tr}\left(\rho a^{1 / 2} b a^{1 / 2}\right)}{\operatorname{tr}(\rho a)}
$$

whenever $\mathcal{P}_{\rho}(a) \neq 0$. Although $\mathcal{P}_{\rho}(\bullet \mid a)$ satisfies the above three conditions for a probability measure, it does not satisfy Bayes' rules. Bayes' first rule says that if $a_{1}+a_{2}+\cdots+a_{n}=I$, then for all $b \in \mathcal{E}(H)$ we have that

$$
\mathcal{P}_{\rho}(b)=\sum_{i=1}^{n} \mathcal{P}\left(a_{i}\right) \mathcal{P}_{\rho}\left(b \mid a_{i}\right)
$$

If (2.1) holds for all $\rho \in \mathcal{S}(H)$ it follows that $b=\sum a_{i} \circ b$. But according to Theorem 1.2(i) this does not hold, in general.

Bayes' second rule says that

$$
\mathcal{P}_{\rho}(b) \mathcal{P}_{\rho}(a \mid b)=\mathcal{P}_{\rho}(a) \mathcal{P}_{\rho}(b \mid a)
$$

Now (2.2) is equivalent to $\mathcal{P}_{\rho}(a \circ b)=\mathcal{P}_{\rho}(b \circ a)$. If this holds for all $\rho \in \mathcal{S}(H)$, it follows that $a b=b a$. Hence, Bayes' second rule does not hold, in general. When $a$ and $b$ are atomic, Theorem 1.2 (ii) characterizes the $\rho \in \mathcal{L}(H)$ for which Bayes' second rule holds.

In a similar way, for $\mathcal{I}, \mathcal{J} \in \mathcal{O}(H)$ we have the conditional probability

$$
\mathcal{P}_{\rho}(\mathcal{J} \mid \mathcal{I})=\frac{\mathcal{P}_{\rho}(\mathcal{I} \circ \mathcal{J})}{\mathcal{P}_{\rho}(\mathcal{I})}=\frac{\operatorname{tr}[\mathcal{J}(\mathcal{I}(\rho))]}{\operatorname{tr}[\mathcal{I}(\rho)]}
$$

whenever $\mathcal{P}_{\rho}(\mathcal{I}) \neq 0$. As before, we write $\mathcal{I} \perp \mathcal{J}$ if $\mathcal{I}+\mathcal{J} \in \mathcal{O}(H)$. We then obtain, $0 \leq \mathcal{P}_{\rho}(\mathcal{J} \mid \mathcal{I}) \leq 1, \mathcal{P}_{\rho}(I \mid \mathcal{I})=1$ and more generally, if $\mathcal{J}$ is a channel, then $\mathcal{P}_{\rho}(\mathcal{J} \mid \mathcal{I})=1$. Also, if $\mathcal{J} \perp \mathcal{K}$, then $\mathcal{P}_{\rho}((\mathcal{J}+\mathcal{K}) \mid \mathcal{I})=$ $\mathcal{P}_{\rho}(\mathcal{J} \mid \mathcal{I})+\mathcal{P}_{\rho}(\mathcal{K} \mid \mathcal{I})$. Similar to $\mathcal{E}(H)$, Bayes' first rule says that if $\mathcal{I}_{1}+\mathcal{I}_{2}+\cdots+\mathcal{I}_{n}=\mathcal{C}$, where $\mathcal{C}$ is a channel, then

$$
\mathcal{P}(\mathcal{J})=\sum_{i=1}^{n} \mathcal{P}_{\rho}\left(\mathcal{I}_{i}\right) \mathcal{P}_{\rho}\left(\mathcal{J} \mid \mathcal{I}_{i}\right)
$$


Notice that we used an arbitrary channel instead of just the trivial channel $I(\rho)=\rho$. The reason for this is that $\mathcal{C}$ corresponds to a certain event because $\mathcal{P}_{\rho}(\mathcal{C})=\operatorname{tr}[\mathcal{C}(\rho)]=1$ for all $\rho \in \mathcal{S}(H)$. Now (2.3) is equivalent to

$$
\begin{aligned}
\operatorname{tr}[\mathcal{J}(\rho)] & =\mathcal{P}_{\rho}(\mathcal{J})=\sum_{i=1}^{n} \mathcal{P}_{\rho}\left[\mathcal{I}_{i} \circ \mathcal{J}(\rho)\right]=\sum_{i=1}^{n} \mathcal{P}_{\rho}\left[\mathcal{J}\left(\mathcal{I}_{i}(\rho)\right)\right] \\
& =\mathcal{P}_{\rho}\left[\mathcal{J}\left(\sum_{i=1}^{n} \mathcal{I}_{i}(\rho)\right)\right]=\mathcal{P}_{\rho}[\mathcal{J}(\mathcal{C}(\rho))]=\operatorname{tr}[\mathcal{J}(\mathcal{C}(\rho))]
\end{aligned}
$$

If $\mathcal{C}=I$ the trivial channel, then clearly (2.4) holds. However as we shall see in later examples, (2.4) does not hold in general.

For the purpose of examples and to better understand the structure of operations, we now consider some special types of operations. An operation $\mathcal{I}$ is sharp if it has the form $\mathcal{I}(\rho)=\sum a_{i} \rho a_{i}$ where $a_{i}$ are projections and $\mathcal{I}$ is atomic if it is sharp and the $a_{i}$ are one-dimensional projections. As in the proof of Theorem [1.2(i), if $\mathcal{I}(\rho)=\sum a_{i} \rho a_{i}$ is sharp then $a_{i} a_{j}=0$ for $i \neq j$ so $\left\{a_{i}\right\}$ are mutually orthogonal projections. We say that $\mathcal{I} \in \mathcal{O}(H)$ is Kraus [10] if $\mathcal{I}(\rho)=A \rho A^{*}$ for some $A \in \mathcal{L}(H)$ with $A^{*} A \leq I$ and $\mathcal{I}$ is Lüders [12] if $\mathcal{I}(\rho)=a \circ \rho=a^{1 / 2} \rho a^{1 / 2}$ for some $a \in \mathcal{E}(H)$. An operation $\mathcal{I}$ is semi-trivial if it has the form $\mathcal{I}(\rho)=\sum \operatorname{tr}\left(\rho a_{i}\right) \alpha_{i}$ where $\alpha_{i} \in \mathcal{S}(H)$ and $a_{i} \in \mathcal{E}(H)$ with $\sum a_{i} \leq I$. Notice that this $\mathcal{I}$ is indeed an operation because

$$
\operatorname{tr}[\mathcal{I}(\rho)]=\operatorname{tr}\left(\rho \sum a_{i}\right) \leq \operatorname{tr}(\rho)
$$

To be specific, we say that $\mathcal{I}$ is semi-trivial with states $\alpha_{i}$ and effects $a_{i}$, $i=1,2, \ldots, n$. An operation $\mathcal{I}$ is trivial if $\mathcal{I}$ is semi-trivial with one state $\alpha$ and one effect $a$. In this case $\mathcal{I}(\rho)=\operatorname{tr}(\rho a) \alpha$ for all $\rho \in \mathcal{S}(H)[8$.

If $\psi \in H$ is a unit vector we denote its corresponding projection operator by $P_{\psi}=|\psi\rangle\langle\psi|$.

Lemma 2.1. An operation $\mathcal{I}$ is atomic if and only if $\mathcal{I}$ is semi-trivial with one-dimensional states $P_{\psi_{i}}$ and corresponding effects $P_{\psi_{i}}$

Proof. We have that $\mathcal{I}$ is atomic if and only if there exists one-dimensional projections $P_{\psi_{i}}$ such that $\mathcal{I}(\rho)=\sum P_{\psi_{i}} \rho P_{\psi_{i}}$. But this is equivalent to

$$
\mathcal{I}(\rho)=\sum\left|\psi_{i}\right\rangle\left\langle\psi_{i}|\rho| \psi_{i}\right\rangle\left\langle\psi_{i}\left|=\sum\left\langle\psi_{i}, \rho \psi_{i}\right\rangle\right| \psi_{i}\right\rangle\left\langle\psi_{i}\right|=\sum \operatorname{tr}\left(\rho P_{\psi_{i}}\right) P_{\psi_{i}}
$$

Now (2.5) is equivalent to $\mathcal{I}$ being semi-trivial with states $P_{\psi_{i}}$ and corresponding effects $P_{\psi_{i}}$. 
Although the Kraus operators for Kraus and Lüders operations are obvious, this is not clear at all for semi-trivial or even trivial operations. This is treated in the next result.

Theorem 2.2. Let $\mathcal{I}(\rho)=\sum_{i=1}^{n} \operatorname{tr}\left(\rho a_{i}\right) \alpha_{i}$ be semi-trivial and let $\alpha_{i}$ have the spectral representation

$$
\alpha_{i}=\sum_{j=1}^{n_{i}} \lambda_{i j}\left|\phi_{i j}\right\rangle\left\langle\phi_{i j}\right|
$$

for $i=1,2, \ldots, n$. Letting $A_{i j k}=\lambda_{i j}^{1 / 2}\left|\phi_{i j}\right\rangle\left\langle a_{i}^{1 / 2} \phi_{i k}\right|$, we have that $\left\{A_{i j k}\right\}$ is a set of Kraus operators for $\mathcal{I}, i=1,2, \ldots, n, j, k=1,2, \ldots, n_{i}$. Moreover, we have that $\widehat{\mathcal{I}}=\sum_{i=1}^{n} a_{i}$.

Proof. Since (2.6) is a spectral representation, we conclude that $\left\{\phi_{i j}: j=1,2, \ldots n_{i}\right\}$ is an orthonormal basis for $H, i=1,2, \ldots, n$ and $\lambda_{i j} \geq 0$ with $\sum_{j=1}^{n_{i}} \lambda_{i j}=1$. Summing over all applicable $i, j, k$, we obtain for all $\rho \in \mathcal{S}(H)$ that

$$
\begin{aligned}
\sum_{i, j, k} A_{i j k} \rho A_{i j k}^{*} & =\sum_{i, j, k} \lambda_{i j}\left|\phi_{i j}\right\rangle\left\langle a_{i}^{1 / 2} \phi_{i k}|\rho| a_{i}^{1 / 2} \phi_{i k}\right\rangle\left\langle\phi_{i j}\right| \\
& =\sum_{i, j, k} \lambda_{i j}\left\langle a_{i}^{1 / 2} \phi_{i k}, \rho a_{i}^{1 / 2} \phi_{i k}\right\rangle\left|\phi_{i j}\right\rangle\left\langle\phi_{i j}\right| \\
& =\sum_{i, j, k} \lambda_{i j}\left\langle\phi_{i k}, a_{i}^{1 / 2} \rho a_{i}^{1 / 2} \phi_{i k}\right\rangle\left|\phi_{i j}\right\rangle\left\langle\phi_{i j}\right| \\
& =\sum_{i, j} \lambda_{i j} \operatorname{tr}\left(\rho a_{i}\right)\left|\phi_{i j}\right\rangle\left\langle\phi_{i j}\right|=\sum_{i} \operatorname{tr}\left(\rho a_{i}\right) \alpha_{i}=\mathcal{I}(\rho)
\end{aligned}
$$

We conclude that

$$
\begin{aligned}
\widehat{\mathcal{I}}=\sum_{i, j, k} A_{i j k}^{*} A_{i j k} & =\sum_{i, j, k} \lambda_{i j}\left|a_{i}^{1 / 2} \phi_{i k}\right\rangle\left\langle\phi_{i j}|| \phi_{i j}\right\rangle\left\langle a_{i}^{1 / 2} \phi_{i k}\right| \\
& =\sum_{i, j, k} \lambda_{i j}\left|a_{i}^{1 / 2} \phi_{i k}\right\rangle\left\langle a_{i}^{1 / 2} \phi_{i k}\left|=\sum_{i, k}\right| a_{i}^{1 / 2} \phi_{i k}\right\rangle\left\langle a_{i}^{1 / 2} \phi_{i k}\right| \\
& =\sum_{i, k} a_{i}^{1 / 2}\left|\phi_{i k}\right\rangle\left\langle\phi_{i k}\right| a_{i}^{1 / 2}=\sum_{i} a_{i}
\end{aligned}
$$

Corollary 2.3. If $\mathcal{I}(\rho)=\operatorname{tr}(\rho a) \alpha$ is a trivial operation and $\alpha$ has spectral representation $\alpha=\sum_{i=1}^{n} \lambda_{i}\left|\phi_{i}\right\rangle\left\langle\phi_{i}\right|$, then $A_{i j}=\lambda_{i}^{1 / 2}\left|\phi_{i}\right\rangle\left\langle a^{1 / 2} \phi_{j}\right|, i, j=$ 
$1.2 \ldots, n$, gives a set of Kraus operators for $\mathcal{I}$. Moreover, we have that $\widehat{\mathcal{I}}=a$.

It can be shown directly that $\widehat{\mathcal{I}}=\sum a_{i}$ in Theorem 2.2. Indeed, we have that

$$
\operatorname{tr}[\mathcal{I}(\rho)]=\sum \operatorname{tr}\left(\rho a_{i}\right)=\operatorname{tr}\left[\rho \sum a_{i}\right]
$$

Moreover, Corollary 2.3 shows that the trivial operation $\mathcal{I}(\rho)=\operatorname{tr}(\rho a) \alpha$ measures the effect $a$. Letting $\alpha$ vary, we obtain an infinite number of different operations that measure $a$. Also, the Lüders operation $\mathcal{L}^{a}(\rho)=$ $a^{1 / 2} \rho a^{1 / 2}$ measures $a$ because

$$
\operatorname{tr}\left[\mathcal{L}^{a}(\rho)\right]=\operatorname{tr}\left(a^{1 / 2} \rho a^{1 / 2}\right)=\operatorname{tr}(\rho a)
$$

for all $\rho \in \mathcal{S}(H)$. We now employ these special operations to show that Bayes' rules do not hold for operations.

Example 1. Let $0<a<I, \alpha \in \mathcal{S}(H)$ and let $\mathcal{I}_{1}=\operatorname{tr}(\rho a) \alpha$ be a trivial operation. Also, let $\mathcal{I}_{2}$ be the trivial operation

$$
\mathcal{I}_{2}(\rho)=\operatorname{tr}\left(\rho a^{\prime}\right) \alpha=[1-\operatorname{tr}(\rho \alpha)] \alpha
$$

Then $\mathcal{C}(\rho)=\left(\mathcal{I}_{1}+\mathcal{I}_{2}\right)(\rho)=\alpha$ is a constant channel. Now (2.4) becomes

$$
\operatorname{tr}[\mathcal{J}(\rho)]=\operatorname{tr}[\mathcal{J}(\mathcal{C}(\rho))]=\operatorname{tr}[\mathcal{J}(\alpha)]
$$

Equation (2.7) does not hold, in general. For example, letting $\mathcal{J}=\mathcal{I}_{1}$, (2.7) becomes

$$
\operatorname{tr}(\rho a)=\operatorname{tr}\left[\mathcal{I}_{1}(\rho)\right]=\operatorname{tr}\left[\mathcal{I}_{1}(\alpha)\right]=\operatorname{tr}(\alpha a)
$$

If $\rho \neq \alpha$, then there exists an $a \in \mathcal{E}(H)$ for which (2.8) fails.

We now give another example for which (2.4) fails.

Example 2. Let $a$ be a projection and define sharp Lüders operations $\mathcal{I}_{1}(\rho)=a \rho a, \mathcal{I}_{2}(\rho)=a^{\prime} \rho a^{\prime}$. Then $\mathcal{C}=\mathcal{I}_{1}+\mathcal{I}_{2}$ is a channel because for all $\rho \in \mathcal{S}(H)$ we obtain

$$
\operatorname{tr}[\mathcal{C}(\rho)]=\operatorname{tr}\left(a \rho a+a^{\prime} \rho a^{\prime}\right)=\operatorname{tr}\left(\rho a+\rho a^{\prime}\right)=\operatorname{tr}(\rho)=1
$$


Let $b \in \mathcal{E}(H)$ and define the Lüders operation $\mathcal{J}(\rho)=b \circ \rho=b^{1 / 2} \rho b^{1 / 2}$.

Then (2.4) becomes

$$
\begin{aligned}
\operatorname{tr}(\rho b) & =\operatorname{tr}[\mathcal{J}(\rho)]=\operatorname{tr}[\mathcal{J}(\mathcal{C}(\rho))]=\operatorname{tr}[\mathcal{J}(a \rho a)]+\operatorname{tr}\left[\mathcal{J}\left(a^{\prime} \rho a^{\prime}\right)\right] \\
& =\operatorname{tr}\left(b^{1 / 2} a \rho a b^{1 / 2}\right)+\left(b^{1 / 2} a^{\prime} \rho a^{\prime} b^{1 / 2}\right)=\operatorname{tr}(\rho a b a)+\operatorname{tr}\left(\rho a^{\prime} b a^{\prime}\right) \\
& =\operatorname{tr}\left[\rho\left(a b a+a^{\prime} b a^{\prime}\right)\right]
\end{aligned}
$$

Now (2.9) holds for all $\rho \in \mathcal{S}(H)$ if and only if $b=a b a+a^{\prime} b a^{\prime}$. Hence, $a b=a b a=b a$. Thus, if $a$ and $b$ do not commute, then (2.9) does not hold for all $\rho \in \mathcal{S}(H)$.

As for effects, Bayes' second rule for operations becomes $\mathcal{P}_{\rho}(\mathcal{I} \circ \mathcal{J})=$ $\mathcal{P}_{\rho}(\mathcal{J} \circ \mathcal{I})$. This is equivalent to

$$
\operatorname{tr}[\mathcal{J}(\mathcal{I}(\rho))]=\operatorname{tr}[\mathcal{I}(\mathcal{J}(\rho))]
$$

Example 3. Let $\mathcal{I}(\rho)=\operatorname{tr}(\rho a) \alpha$ and $\mathcal{J}(\rho)=\operatorname{tr}(\rho a) \beta$ be trivial operations. Then

$$
\operatorname{tr}[\mathcal{J}(\mathcal{I}(\rho))]=\operatorname{tr}(\rho a) \operatorname{tr}[\mathcal{J}(\alpha)]=\operatorname{tr}(\rho a) \operatorname{tr}(\alpha a)
$$

and similarly, $\operatorname{tr}[\mathcal{I}(\mathcal{J}(\rho))]=\operatorname{tr}(\rho a) \operatorname{tr}(\beta a)$. These are not the same so (2.10) fails, in general. As another example, let $\mathcal{I}(\rho)=a^{1 / 2} \rho a^{1 / 2}$, $\mathcal{J}(\rho)=b^{1 / 2} \rho b^{1 / 2}$ be Lüders operations. We then obtain

$$
\left.\operatorname{tr}[\mathcal{J}(\mathcal{I}(\rho))]=\operatorname{tr}\left(b^{1 / 2} a^{1 / 2} \rho a^{1 / 2} b^{1 / 2}\right)=\operatorname{tr}(\rho a \circ b)\right]
$$

and similarly, $\operatorname{tr}[\mathcal{I}(\mathcal{J}(\rho))]=\operatorname{tr}(\rho b \circ a)$. These are equal if and only if $a b=b a$ so again(2.10) fails, in general.

We close this section by considering complements of operations. For $\mathcal{I}, \mathcal{J} \in \mathcal{O}(H)$, we write $\mathcal{I} \leq \mathcal{J}$ if $\mathcal{I}(\rho) \leq \mathcal{J}(\rho)$ for all $\rho \in \mathcal{S}(H)$. If $\mathcal{I} \leq \mathcal{C}$ where $\mathcal{C}$ is a channel, we call $\mathcal{I}^{\mathcal{C}}=\mathcal{C}-\mathcal{I}$ the $\mathcal{C}$-complement of $\mathcal{I}$. Then $\mathcal{I}^{\mathcal{C}}$ is the unique operation satisfying $\mathcal{I}+\mathcal{I}^{\mathcal{C}}=\mathcal{C}$. An operation other than $I$ can have many complements. For example, any channel is a complement of 0 . We frequently say, for short, that $\mathcal{J}$ is a complement of $\mathcal{I}$ instead of $\mathcal{J}$ is a $\mathcal{C}$-complement of $\mathcal{I}$. 
Example 4. Let $\mathcal{I} \in \mathcal{O}(H)$ with Kraus decomposition $\mathcal{I}(\rho)=\sum A_{i} \rho A_{i}^{*}$. Letting $b=\sum A_{i}^{*} A_{i}$ we have that $b \in \mathcal{E}(H)$. Then the Lüders operation $\mathcal{J}(\rho)=(I-b)^{1 / 2} \rho(I-b)^{1 / 2}$ is a complement of $\mathcal{I}$ because

$$
(\mathcal{I}+\mathcal{J})(\rho)=\mathcal{I}(\rho)+\mathcal{J}(\rho)=\sum A_{i} \rho A_{i}^{*}+(I-b)^{1 / 2} \rho(I-b)^{1 / 2}
$$

and since $\sum A_{i}^{*} A_{i}+I-b=I$, we conclude that $\mathcal{I}+\mathcal{J}$ is a channel. This shows that any operation has a unique Lüders operation complement.

Example 5. Let $\mathcal{I}(\rho)=a \circ \rho=a^{1 / 2} \rho a^{1 / 2}$ be a Lüders operation with $a \in \mathcal{E}(H)$. Then $\mathcal{J}(\rho)=a^{\prime} \circ \rho$ is a complement of $\mathcal{I}$ because $\mathcal{I}(\rho)+\mathcal{J}(\rho)=a \circ \rho+a^{\prime} \circ \rho$ is a channel. Moreover, if $\mathcal{I}(\rho)=\operatorname{tr}(\rho a) \alpha$ is trivial, then $\mathcal{J}(\rho)=\operatorname{tr}\left(\rho a^{\prime}\right) \alpha$ is a complement of $\mathcal{I}$ because

$$
(\mathcal{I}+\mathcal{J})(\rho)=\mathcal{I}(\rho)+\mathcal{J}(\rho)=\operatorname{tr}(\rho a) \alpha+\operatorname{tr}\left(\rho a^{\prime}\right) \alpha=\operatorname{tr}(\rho) \alpha=\alpha
$$

is a channel.

Theorem 2.4. (i) $\mathcal{J}$ is a complement of $\mathcal{I}$ if and only if $\widehat{\mathcal{J}}=(\mathcal{I})^{\prime}$. (ii) If $\mathcal{I}$ is sharp, then $\mathcal{I} \wedge \mathcal{I}^{\mathcal{C}}=0$ for some channel $\mathcal{C}$.

Proof. (i) Suppose that $\mathcal{J}$ is a $\mathcal{C}$-complement of $\mathcal{I}$ so $\mathcal{J}=\mathcal{I}^{\mathcal{C}}=\mathcal{C}-\mathcal{I}$. Then

$$
\widehat{\mathcal{J}}=\widehat{\mathcal{C}}-\widehat{\mathcal{I}}=I-\widehat{\mathcal{I}}=(\widehat{\mathcal{I}})^{\prime}
$$

Conversely, if $\widehat{\mathcal{J}}=(\widehat{\mathcal{I}})^{\prime}$ then

$$
\operatorname{tr}[\mathcal{J}(\rho)+\mathcal{I}(\rho)]=\operatorname{tr}(\rho \mathcal{J})+\operatorname{tr}(\rho \widehat{\mathcal{I}})=\operatorname{tr}(\rho)=1
$$

for all $\rho \in \mathcal{S}(H)$. Hence, $\mathcal{J}+\mathcal{I}$ is a channel so $\mathcal{J}$ is a complement of $\mathcal{I}$. (ii) . Let $\mathcal{I}(\rho)=\sum a_{i} \rho a_{i}$ where $a_{i}$ are projections and let $\mathcal{J}(\rho)=b^{\prime} \rho b^{\prime}$ where $b=\sum a_{i}$. Then letting

$$
\mathcal{C}(\rho)=\mathcal{I}(\rho)+\mathcal{J}(\rho)=\sum a_{i} \rho a_{i}+b^{\prime} \rho b^{\prime}
$$

we have that

$$
\operatorname{tr}[\mathcal{C}(\rho)]=\operatorname{tr}\left[\rho\left(\sum a_{i}+b^{\prime}\right)\right]=\operatorname{tr}(\rho)=1
$$

for every $\rho \in \mathcal{S}(H)$ so $\mathcal{C}$ is a channel. Hence, $\mathcal{J}=\mathcal{I}^{\mathcal{C}}$. To show that $\mathcal{I} \wedge \mathcal{J}=0$, let $\mathcal{K} \leq \mathcal{I}, \mathcal{J}$. Then $\widehat{\mathcal{K}} \leq \widehat{\mathcal{I}}=\sum a_{i}$ and

$$
\widehat{\mathcal{K}} \leq \widehat{\mathcal{J}}=b^{\prime}=I-\sum a_{i}
$$

It follows that $\widehat{\mathcal{K}}=0$ so $\mathcal{K}=0$. 


\section{Observables and Instruments}

We now extend our previous work to observables and instruments. An observable is a finite set $A=\left\{a_{x}: x \in \Omega_{A}\right\} \subseteq \mathcal{E}(H)$ satisfying $\sum_{x \in \Omega_{A}} a_{x}=I$ [1, 2, 8, 13. We call $\Omega_{A}$ the outcome set and $x \in \Omega_{A}$ is an outcome for $A$. We think of $A$ as an experiment with possible outcomes $x \in \Omega_{A}$ and $a_{x}$ is the effect that is yes when $A$ has outcome $x$. The probability that $A$ has outcome $x$ when the system is in state $\rho \in \mathcal{S}(H)$ is $\mathcal{P}_{\rho}(x)=\operatorname{tr}\left(\rho a_{x}\right)$ and we call $\Phi_{\rho}^{A}(x)=\mathcal{P}_{\rho}(x)$ the distribution of $A$. If $\Delta \subseteq \Omega_{A}$, we define the probability of $\Delta$ in the state $\rho$ by

$$
\Phi_{\rho}^{A}(\Delta)=\mathcal{P}_{\rho}(\Delta)=\sum\left\{\mathcal{P}_{\rho}(x): x \in \Delta\right\}=\sum\left\{\operatorname{tr}\left(\rho a_{x}\right): x \in \Delta\right\}
$$

We see that $\Delta \mapsto \sum_{x \in \Delta} A_{x}$ is an effect-valued measure on $2^{\Omega_{A}}$. We denote the set of observables on $H$ by $\mathrm{Ob}(H)$.

If $A=\left\{a_{x}: x \in \Omega_{A}\right\}$ and $B=\left\{b_{y}: y \in \Omega_{B}\right\}$ are observables, we define their sequential product [5, 6, 7]

$$
(A \circ B)_{(x, y)}=\left\{a_{x} \circ b_{y}:(x, y) \in \Omega_{A} \times \Omega_{B}\right\}
$$

with outcome set $\Omega_{A \circ B}=\Omega_{A} \times \Omega_{B}$. Notice that $A \circ B$ is indeed an observable because

$$
\sum_{(x, y) \in \Omega_{A \circ B}}(A \circ B)_{(x, y)}=\sum_{x, y}\left(a_{x} \circ b_{y}\right)=\sum_{x} a_{x} \circ \sum_{y} b_{y}=\sum_{x} a_{x}=I
$$

We also have the observable $B$ conditioned by the observable $A$ defined as [5]

$$
(B \mid A)_{y}=\sum_{x \in \Omega_{A}}(A \circ B)_{(x, y)}=\sum_{x \in \Omega_{A}}\left(a_{x} \circ b_{y}\right)
$$

where $\Omega_{(B \mid A)}=\Omega_{B}$. Again, $(B \mid A)$ is an observable because

$$
\sum_{y \in \Omega_{B}}(B \mid A)_{y}=\sum_{x, y}\left(a_{x} \circ b_{y}\right)=I
$$

Just as for effects, Bayes' rule

$$
\mathcal{P}_{\rho}\left(b_{y}\right)=\sum_{x} \mathcal{P}_{\rho}\left(a_{x}\right) \mathcal{P}_{\rho}\left(b_{y} \mid a_{x}\right)
$$


does not hold. However, we do have the result

$$
\mathcal{P}_{\rho}\left[(B \mid A)_{y}\right]=\sum_{x} \mathcal{P}_{\rho}\left(a_{x}\right) \mathcal{P}_{\rho}\left(b_{y} \mid a_{x}\right)
$$

Indeed, for all $\rho \in \mathcal{S}(H)$ we obtain

$$
\begin{aligned}
\mathcal{P}_{\rho}\left[(B \mid A)_{y}\right] & =\operatorname{tr}\left[\rho(B \mid A)_{y}\right]=\operatorname{tr}\left[\rho \sum_{x}\left(a_{x} \circ b_{y}\right)\right]=\sum_{x} \operatorname{tr}\left(\rho a_{x} \circ b_{y}\right) \\
& =\sum_{x} \operatorname{tr}\left(a_{x}^{1 / 2} \rho a_{x}^{1 / 2} b_{y}\right)=\sum_{x} \operatorname{tr}\left(\rho a_{x}\right) \mathcal{P}_{\rho}\left(b_{y} \mid a_{x}\right) \\
& =\sum_{x} \mathcal{P}_{\rho}\left(a_{x}\right) \mathcal{P}_{\rho}\left(b_{y} \mid a_{x}\right)
\end{aligned}
$$

Also, notice that $y \mapsto \mathcal{P}_{\rho}\left(b_{y} \mid a_{x}\right)$ is additive so it is a real-valued measure.

An instrument [1, 8, 13] is a finite set $\mathcal{I}=\left\{\mathcal{I}_{x}: x \in \Omega_{\mathcal{I}}\right\} \subseteq \mathcal{O}(H)$ satisfying $\overline{\mathcal{I}}=\sum_{x \in \Omega_{\mathcal{I}}} \mathcal{I}_{x}$ is a channel. We call $\Omega_{\mathcal{I}}$ the outcome set and $x \in \Omega_{\mathcal{I}}$ is an outcome for $\mathcal{I}$. The probability that $\mathcal{I}$ has outcome $x$ when the system is in state $\rho \in \mathcal{S}(H)$ is $\mathcal{P}_{\rho}(x)=\operatorname{tr}\left[\mathcal{I}_{x}(\rho)\right]$ and we call $\Phi_{\rho}^{\mathcal{I}}(x)=\mathcal{P}_{\rho}(x)$ the distribution of $\mathcal{I}$. If $\Delta \subseteq \Omega_{\mathcal{I}}$, we define the probability of $\Delta$ in the state $\rho$ by

$$
\Phi_{\rho}^{\mathcal{I}}(\Delta)=\mathcal{P}_{\rho}(\Delta)=\sum\left\{\mathcal{P}_{\rho}(x): x \in \Delta\right\}=\sum\left\{\operatorname{tr}\left[\mathcal{I}_{x}(\rho)\right]: x \in \Delta\right\}
$$

Then $\Delta \mapsto \sum_{x \in \Delta} \mathcal{I}_{x}$ is an operation-valued measure on $2^{\Omega_{\mathcal{I}}}$. We denote the set of instruments on $H$ by $\operatorname{In}(H)$. We say that $\mathcal{I} \in \operatorname{In}(H)$ measures $A \in \mathrm{Ob}(H)$ if $\Omega_{A}=\Omega_{\mathcal{I}}$ and $\Phi_{\rho}^{A}(x)=\Phi_{\rho}^{\mathcal{I}}(x)$ for all $x \in \Omega_{A}, \rho \in \mathcal{S}(H)$. Since this is equivalent to $\widehat{\mathcal{I}}_{x}=a_{x}$, we have that $\mathcal{I}$ measures a unique $\widehat{\mathcal{I}} \in \mathrm{Ob}(H)$ given by $\widehat{\mathcal{I}}=\left\{\widehat{\mathcal{I}}_{x}: x \in \Omega_{\mathcal{I}}\right\}$. We think of $\mathcal{I}$ as an apparatus that is employed to measure the observable $\widehat{\mathcal{I}}$. Although $\mathcal{I}$ measures the unique $\widehat{\mathcal{I}} \in \mathrm{Ob}(H)$, as we shall see, an observable is measured by many instruments.

If $\mathcal{I}=\left\{\mathcal{I}_{x}: x \in \Omega_{\mathcal{I}}\right\}$ and $\mathcal{J}=\left\{\mathcal{J}_{y}: y \in \Omega_{\mathcal{J}}\right\}$ are instruments, we define their sequential product [5, 6, 7]

$$
(\mathcal{I} \circ \mathcal{J})_{(x, y)}=\left\{\mathcal{I}_{x} \circ \mathcal{J}_{y}:(x, y) \in \Omega_{\mathcal{I}} \times \Omega_{\mathcal{J}}\right\}
$$

with outcome set $\Omega_{\mathcal{I} \circ \mathcal{J}}=\Omega_{\mathcal{I}} \times \Omega_{\mathcal{J}}$. We see that $\mathcal{I} \circ \mathcal{J}$ is indeed an instrument because

$$
\sum_{(x, y) \in \Omega_{\mathcal{I} \circ \mathcal{J}}}(\mathcal{I} \circ \mathcal{J})_{(x, y)}=\sum_{x, y} \mathcal{I}_{x} \circ \mathcal{J}_{y}=\sum_{x} \mathcal{I}_{x} \circ \sum_{y} \mathcal{J}_{y}=\overline{\mathcal{I}} \circ \overline{\mathcal{J}}
$$


which is a channel. We also have the instrument $\mathcal{J}$ conditioned by $\mathcal{I}$ defined as

$(\mathcal{J} \mid \mathcal{I})_{y}(\rho)=\sum_{x}\left(\mathcal{I}_{x} \circ \mathcal{J}_{y}\right)(\rho)=\sum_{x} \mathcal{J}_{y}\left[\mathcal{I}_{x}(\rho)\right]=\mathcal{J}_{y}\left[\sum_{x} \mathcal{I}_{x}(\rho)\right]=\mathcal{J}_{y}[\overline{\mathcal{I}}(\rho)]$

We have that $(\mathcal{J} \mid \mathcal{I})$ is indeed an instrument with outcome space $\Omega_{\mathcal{J}}$ because

$$
\sum_{y}(\mathcal{J} \mid \mathcal{I})_{y}=\sum_{y} \mathcal{J}_{y}[\overline{\mathcal{I}}(\rho)]=\overline{\mathcal{J}}[\overline{\mathcal{I}}(\rho)]
$$

which is a channel. As with observables, we have that

$$
\mathcal{P}_{\rho}\left[(\mathcal{J} \mid \mathcal{I})_{y}\right]=\sum_{x} \mathcal{P}_{\rho}\left(\mathcal{I}_{x}\right) \mathcal{P}_{\rho}\left(\mathcal{J}_{y} \mid \mathcal{I}_{x}\right)
$$

because

$$
\begin{aligned}
\mathcal{P}_{\rho}\left[(\mathcal{J} \mid \mathcal{I})_{y}\right] & =\operatorname{tr}\left[(\mathcal{J} \mid \mathcal{I})_{y}\right]=\operatorname{tr}\left\{\sum_{x} \mathcal{J}_{y}\left[\mathcal{I}_{x}(\rho)\right]\right\} \\
& =\sum_{x} \operatorname{tr}\left\{\left[\mathcal{I}_{x}(\rho)\right] \mathcal{P}_{\rho}\left(\mathcal{J}_{y} \mid \mathcal{I}_{x}\right)\right\}=\sum_{x} \mathcal{P}_{\rho}\left(\mathcal{I}_{x}\right) \mathcal{P}_{\rho}\left(\mathcal{J}_{y} \mid \mathcal{I}_{x}\right)
\end{aligned}
$$

Also notice that $y \mapsto \mathcal{P}_{\rho}\left(\mathcal{J}_{y} \mid \mathcal{I}_{x}\right)$ is additive and a probability measure because

$$
\begin{aligned}
\sum_{y} \mathcal{P}_{\rho}\left(\mathcal{J}_{y} \mid \mathcal{I}_{x}\right) & =\frac{1}{\operatorname{tr}\left[\mathcal{I}_{x}(\rho)\right]} \operatorname{tr}\left\{\sum_{y} \mathcal{J}_{y}\left[\mathcal{I}_{x}(\rho)\right]\right\}=\frac{1}{\operatorname{tr}\left[\mathcal{I}_{x}(\rho)\right]} \operatorname{tr}\left[\overline{\mathcal{J}}\left(\mathcal{I}_{x}(\rho)\right)\right] \\
& =\operatorname{tr}\left[\overline{\mathcal{J}}\left(\frac{\mathcal{I}_{x}(\rho)}{\operatorname{tr}\left(\mathcal{I}_{x}(\rho)\right)}\right)\right]=1
\end{aligned}
$$

As with operations, Bayes' rules do not hold for instruments.

We now consider various types of instruments. A general instrument $\mathcal{I}=\left\{\mathcal{I}_{x}: x \in \Omega_{\mathcal{I}}\right\}$ has a Kraus decomposition $\mathcal{I}_{x}(\rho)=\sum_{i=1}^{n_{x}} A_{i}^{x} \rho\left(A_{i}^{x}\right)^{*}$ with $\widehat{\mathcal{I}}_{x}=\sum_{i=1}^{n_{x}}\left(A_{i}^{x}\right)^{*} A_{i}^{x} \leq I$ and $\overline{\mathcal{I}}(\rho)=\sum_{x} \sum_{i=1}^{n_{x}} A_{i}^{x} \rho\left(A_{i}^{x}\right)^{*}$ with $\sum_{x} \sum_{i=1}^{n_{x}}\left(A_{i}^{x}\right)^{*} A_{i}^{x}=I$. We say that $\mathcal{I}$ is sharp if $A_{i}^{x}$ are projections for all $i, x$. We then have that $\sum_{x, i} A_{i}^{x}=I$ and it follows that $A_{i}^{x} A_{j}^{y}=0$ if $(x, i) \neq(y, j)$. We say that $\mathcal{I}$ is atomic if $A_{i}^{x}$ are one-dimensional projections for all $i, x$. An instrument $\mathcal{I}$ is Kraus [10] if it has Kraus decompositions $\mathcal{I}_{x}(\rho)=A_{x} \rho A_{x}^{*}$ for all $x \in \Omega_{\mathcal{I}}$ 
in which case $\sum_{x} A_{x}^{*} A_{x}=I$ and $\widehat{\mathcal{I}}=\left\{A_{x}^{*} A_{x}: x \in \Omega_{\mathcal{I}}\right\}$. An instrument $\mathcal{I}$ is Lüders [12] if it is Kraus and has decomposition

$$
\mathcal{I}_{x}(\rho)=a_{x} \circ \rho=a_{x}^{1 / 2} \rho a_{x}^{1 / 2}
$$

for every $x \in \Omega_{\mathcal{I}}$ where $a_{x} \in \mathcal{E}(H)$. In this case $\sum_{x} a_{x}=I$ and we obtain the observable $\widehat{\mathcal{I}}=\left\{a_{x}: x \in \Omega_{\mathcal{I}}\right\}$. We then use the notation $A=\widehat{\mathcal{I}}$ and write $\mathcal{I}=\mathcal{L}^{A}$. It follows that $\left(\mathcal{L}^{A}\right)^{\wedge}=A$. An instrument $\mathcal{I}$ is trivial if there exists an observable $A=\left\{a_{x}: x \in \Omega_{\mathcal{I}}\right\}$ and $\alpha \in \mathcal{S}(H)$ such that $\mathcal{I}_{x}(\rho)=\operatorname{tr}\left(\rho a_{x}\right) \alpha$ for all $x \in \Omega_{\mathcal{I}}$. In this case $\mathcal{I}_{x}=a_{x}$ so $\mathcal{I}$ measures $A$. We then say that $\mathcal{I}$ is trivial with observable $A$ and state $\alpha$. More generally, we say that $\mathcal{I}$ is semi-trivial with observable $A=\left\{a_{x}: x \in \Omega_{\mathcal{I}}\right\}$ and states $\alpha_{x}$ if $\mathcal{I}_{x}(\rho)=\operatorname{tr}\left(\rho a_{x}\right) \alpha_{x}$. We again have that $\widehat{\mathcal{I}}_{x}=a_{x}$ so $\mathcal{I}$ measures $A$. These last three types illustrate that an observable can be measured by many different instruments

Theorem 3.1. (i) For any $\mathcal{I}, \mathcal{J} \in \operatorname{In}(H)$ we have

$$
\overline{(\mathcal{I} \circ \mathcal{J})}=\overline{(\mathcal{J} \mid \mathcal{I})}=\overline{\mathcal{I}} \circ \overline{\mathcal{J}}
$$

(ii) If $A \in \mathrm{Ob}(H)$ and $\mathcal{I} \in \operatorname{In}(H)$, then

$$
\left(\mathcal{L}^{A} \circ \mathcal{I}\right)^{\wedge}=\left(\mathcal{L}^{A}\right)^{\wedge} \circ \widehat{\mathcal{I}}=A \circ \widehat{\mathcal{I}}
$$

(iii) If $A, B \in \mathrm{Ob}(H)$, then

$$
\left(\mathcal{L}^{A} \circ \mathcal{L}^{B}\right)^{\wedge}=\left(\mathcal{L}^{A}\right)^{\wedge} \circ\left(\mathcal{L}^{B}\right)^{\wedge}=A \circ B
$$

Proof. (i) We have that

$$
\overline{(\mathcal{I} \circ \mathcal{J})}=\sum_{x, y}(\mathcal{I} \circ \mathcal{J})_{(x, y)}=\sum_{x, y} \mathcal{I}_{x} \circ \mathcal{J}_{y}=\sum_{x} \mathcal{I}_{x} \circ \sum_{y} \mathcal{J}_{y}=\overline{\mathcal{I}} \circ \overline{\mathcal{J}}
$$

Moreover,

$$
\overline{(\mathcal{J} \mid \mathcal{I})}=\sum_{y}(\mathcal{J} \mid \mathcal{I})_{y}=\sum_{y} \sum_{x} \mathcal{I}_{x} \circ \mathcal{J}_{y}=\overline{\mathcal{I}} \circ \overline{\mathcal{J}}
$$

(ii) Letting $A=\left\{a_{x}: x \in \Omega_{A}\right\}$ we obtain

$\operatorname{tr}\left[\rho\left(\mathcal{L}^{A} \circ \mathcal{I}\right)_{(x, y)}^{\wedge}\right]=\operatorname{tr}\left[\left(\mathcal{L}^{A} \circ \mathcal{I}\right)_{(x, y)}(\rho)\right]=\operatorname{tr}\left[\left(\mathcal{L}_{x}^{A} \circ \mathcal{I}_{y}\right)(\rho)\right]$ 


$$
\begin{aligned}
& =\operatorname{tr}\left[\mathcal{I}_{y}\left(a_{x}^{1 / 2} \rho a_{x}^{1 / 2}\right)\right]=\operatorname{tr}\left[a_{x}^{1 / 2} \rho a_{x}^{1 / 2} \widehat{\mathcal{I}}_{y}\right] \\
& =\operatorname{tr}\left(\rho a_{x}^{1 / 2} \widehat{\mathcal{I}}_{y} a_{x}^{1 / 2}\right)=\operatorname{tr}\left(\rho a_{x} \circ \widehat{\mathcal{I}}_{y}\right)=\operatorname{tr}\left[\rho(A \circ \widehat{\mathcal{I}})_{(x, y)}\right]
\end{aligned}
$$

for all $\rho \in \mathcal{S}(H)$. Hence, $\left(\mathcal{L}^{A} \circ \mathcal{I}\right)_{(x, y)}^{\wedge}=(A \circ \widehat{\mathcal{I}})_{(x, y)}$ for all $(x, y) \in \Omega_{A} \times \Omega_{\mathcal{I}}$. We conclude that $\left(\mathcal{L}^{A} \circ \mathcal{I}\right)^{\wedge}=A \circ \widehat{\mathcal{I}}=\left(\mathcal{L}^{A}\right)^{\wedge} \circ \widehat{\mathcal{I}}$. (iii) follows from (ii).

Example 6. Unlike Theorem $3.1\left(\right.$ ii) we show that $\left(\mathcal{I} \circ \mathcal{L}^{A}\right)^{\wedge} \neq \widehat{\mathcal{I}} \circ A$, in general. Let $\mathcal{I}_{x}(\rho)=\operatorname{tr}\left(\rho b_{x}\right) \alpha$ be a trivial instrument. Letting $A=\left\{a_{y}: y \in \Omega_{A}\right\}$ we have

$$
(\widehat{\mathcal{I}} \circ A)_{(x, y)}=\widehat{\mathcal{I}}_{x} \circ a_{y}=b_{x} \circ a_{y}=b_{x}^{1 / 2} a_{y} b_{x}^{1 / 2}
$$

On the other hand, since

$$
\begin{aligned}
\operatorname{tr}\left[\rho\left(\mathcal{I} \circ \mathcal{L}^{A}\right)_{(x, y)}^{\wedge}\right] & =\operatorname{tr}\left[\left(\mathcal{I} \circ \mathcal{L}^{A}\right)_{(x, y)}(\rho)\right]=\operatorname{tr}\left[\left(\mathcal{I}_{x} \circ \mathcal{L}_{y}^{A}\right)(\rho)\right] \\
& =\operatorname{tr}\left[a_{y}^{1 / 2} \mathcal{I}_{x}(\rho) a_{y}^{1 / 2}\right]=\operatorname{tr}\left(\rho b_{x}\right) \operatorname{tr}\left(a_{y}^{1 / 2} \alpha a_{y}^{1 / 2}\right) \\
& =\operatorname{tr}\left(\rho b_{x}\right) \operatorname{tr}\left(\alpha a_{y}\right)=\operatorname{tr}\left[\rho \operatorname{tr}\left(\alpha a_{y}\right) b_{x}\right]
\end{aligned}
$$

we have that $\left(\mathcal{I} \circ \mathcal{L}^{A}\right)_{(x, y)}^{\wedge}=\operatorname{tr}\left(\alpha a_{y}\right) b_{x}$ it is clear that $b_{x}^{1 / 2} a_{y} b_{x}^{1 / 2} \neq \operatorname{tr}\left(\alpha a_{y}\right) b_{x}$, in general.

Example 7. Let $A=\left\{a_{x}: x \in \Omega_{A}\right\}, B=\left\{b_{y}: y \in \Omega_{B}\right\}$ be observable and let $\mathcal{I}_{x}(\rho)=\operatorname{tr}\left(\rho a_{x}\right) \alpha, \mathcal{J}_{y}(\rho)=\operatorname{tr}\left(\rho b_{y}\right) \beta$ be trivial instruments. We show that $(\mathcal{I} \circ \mathcal{J})^{\wedge} \neq \widehat{\mathcal{I}} \circ \widehat{\mathcal{J}}$, in general. We have that

$$
\operatorname{tr}\left[\rho(\mathcal{I} \circ \mathcal{J})_{(x, y)}^{\wedge}\right]=\operatorname{tr}\left[(\mathcal{I} \circ \mathcal{J})_{(x, y)}(\rho)\right]=\operatorname{tr}\left[\mathcal{J}_{y}\left(\mathcal{I}_{x}(\rho)\right)\right]=\operatorname{tr}\left(\rho a_{x}\right) \operatorname{tr}\left(\alpha b_{y}\right)
$$

However, $\operatorname{tr}\left(\rho \widehat{\mathcal{I}}_{x} \circ \widehat{\mathcal{J}}_{y}\right)=\operatorname{tr}\left(\rho a_{x} b_{y}\right)$ and these do not agree, in general. For example, if $a_{x}=|\phi\rangle\left\langle\phi\left|, b_{y}=\right| \psi\right\rangle\langle\psi|$ where $|\langle\phi, \psi\rangle|^{2} \neq\langle\psi, \alpha \psi\rangle$ then

$$
\begin{aligned}
\operatorname{tr}\left[\rho(\mathcal{I} \circ \mathcal{J})_{(x, y)}^{\wedge}\right] & =\langle\phi \rho \phi\rangle\langle\psi, \alpha \psi\rangle \neq|\langle\phi, \psi\rangle|^{2}\langle\phi, \rho \phi\rangle \\
& =|\langle\phi, \psi\rangle|^{2} \operatorname{tr}(\rho|\phi\rangle\langle\phi|)=\operatorname{tr}(\rho|\phi\rangle\langle\phi|| \psi\rangle\langle\psi|| \phi\rangle\langle\phi|) \\
& =\operatorname{tr}\left(\rho a_{x} \circ b_{y}\right)
\end{aligned}
$$

Hence, $\widehat{\mathcal{I}} \circ \widehat{\mathcal{J}}=A \circ B \neq(\mathcal{I} \circ \mathcal{J})^{\wedge}$. 
Example 8. We give another example in which $(\mathcal{I} \circ \mathcal{J})^{\wedge} \neq \widehat{\mathcal{I}} \circ \widehat{\mathcal{J}}$. Let $\mathcal{I}_{x}(\rho)=A_{x} \rho A_{x}^{*}, \mathcal{J}_{y}(\rho)=B_{y} \rho B_{y}^{*}$ be Kraus instruments. Then

$\operatorname{tr}\left[(\mathcal{I} \circ \mathcal{J})_{(x, y)}(\rho)\right]=\operatorname{tr}\left[\mathcal{J}_{y}\left(\mathcal{I}_{x}(\rho)\right)\right]=\operatorname{tr}\left[B_{y}\left(A_{x} \rho A_{x}^{*}\right) B_{y}^{*}\right]=\operatorname{tr}\left(\rho A_{x}^{*} B_{y}^{*} B_{y} A_{x}\right)$

Hence, $(\mathcal{I} \circ \mathcal{J})_{(x, y)}^{\wedge}=A_{x}^{*} B_{y}^{*} B_{y} A_{x}$. On the other hand,

$$
(\widehat{\mathcal{I}} \circ \widehat{\mathcal{J}})_{(x, y)}=\widehat{\mathcal{I}}_{x} \circ \widehat{\mathcal{J}}_{y}=\left(A_{x}^{*} A_{x}\right) \circ\left(B_{y}^{*} B_{y}\right)=\left(A_{x}^{*} A_{x}\right)^{1 / 2} B_{y}^{*} B_{y}\left(A_{x}^{*} A_{x}\right)^{1 / 2}
$$

and these are not equal, in general.

Lemma 3.2. (i) If $\mathcal{I}$ and $\mathcal{J}$ are semi-trivial with states $\alpha_{x}$, observable $A=\left\{a_{x}\right\}$ and states $\beta_{y}$, observable $B=\left\{b_{y}\right\}$, respectively, then $\mathcal{I} \circ \mathcal{J}$ is semi-trivial with states $\beta_{y}$ and observable $\left\{\operatorname{tr}\left(\alpha_{x} b_{y}\right) a_{x}\right\}$. Also, $(\mathcal{J} \mid \mathcal{I})$ is semi-trivial with states $\beta_{y}$ and observable $\left\{\sum_{x} \operatorname{tr}\left(\alpha_{x} \beta_{y}\right) a_{x}\right\}$. (ii) If $\mathcal{I}$ and $\mathcal{J}$ are trivial with state $\alpha$, observable $\left\{a_{x}\right\}$ and state $\beta$, observable $\left\{b_{y}\right\}$, respectively, the $\mathcal{I} \circ \mathcal{J}$ is trivial with state $\beta$ and observable $\left\{\operatorname{tr}\left(\alpha b_{y}\right) a_{x}\right\}$. Also, $(\mathcal{J} \mid \mathcal{I})$ is trivial with state $\beta$ and observable $\left\{\operatorname{tr}\left(\alpha b_{y}\right) I\right\}$.

Proof. (i) Since

$$
\begin{aligned}
(\mathcal{I} \circ \mathcal{J})_{(x, y)}(\rho) & =\mathcal{J}_{y}\left[\mathcal{I}_{x}(\rho)\right]=\mathcal{J}_{y}\left[\operatorname{tr}\left(\rho a_{x}\right) \alpha_{x}\right]=\operatorname{tr}\left(\rho a_{x}\right) \operatorname{tr}\left(\alpha_{x} b_{y}\right) \beta_{y} \\
& =\operatorname{tr}\left[\rho \operatorname{tr}\left(\alpha_{x} b_{y}\right) a_{x}\right] \beta_{y}
\end{aligned}
$$

We conclude that $\mathcal{I} \circ \mathcal{J}$ is semi-trivial with states $\beta_{y}$ and observable $\operatorname{tr}\left(\alpha_{x} b_{y}\right) a_{x}$. Moreover, since

$$
(\mathcal{J} \mid \mathcal{I})_{y}(\rho)=\mathcal{J}_{y}[\overline{\mathcal{I}}(\rho)]=\mathcal{J}_{y}\left[\sum_{x} \operatorname{tr}\left(\rho a_{x}\right) \alpha_{x}\right]=\operatorname{tr}\left[\rho \sum_{x} \operatorname{tr}\left(\alpha_{x} b_{y}\right) a_{x}\right] \beta_{y}
$$

we conclude that $(\mathcal{J} \mid \mathcal{I})$ is semi-trivial with states $\beta_{y}$ and observable $\left\{\sum_{x} \operatorname{tr}\left(\alpha_{x} \beta_{y}\right) a_{x}\right\}$. (ii) follows from (i).

We have seen that the sequential product of trivial (semi-trivial) instruments is trivial (semi-trivial). Also, the sequential product of two Kraus instruments $\mathcal{I}_{x}(\rho)=A_{x} \rho A_{x}^{*}$ and $\mathcal{J}_{y}(\rho)=B_{y} \rho B_{y}^{*}$ is Kraus because

$$
\mathcal{I}_{x} \circ \mathcal{J}_{y}(\rho)=\mathcal{J}_{y}\left(\mathcal{I}_{x}(\rho)\right)=B_{y} A_{x} \rho A_{x}^{*} B_{y}^{*}=B_{y} A_{x} \rho\left(B_{y} A_{x}\right)^{*}
$$


so the Kraus operator for $\mathcal{I}_{x} \circ \mathcal{J}_{y}$ is $B_{y} A_{x}$. However, the sequential product of two Lüders instruments $\mathcal{I}_{x}(\rho)=a_{x}^{1 / 2} \rho a_{x}^{1 / 2}, \mathcal{J}_{y}(\rho)=b_{y}^{1 / 2} \rho b_{y}^{1 / 2}$ given by

$$
\mathcal{I} \circ \mathcal{J}_{y}=a_{x}^{1 / 2} b_{y}^{1 / 2} \rho b_{y}^{1 / 2} a_{x}^{1 / 2}
$$

need not be Lüders.

Lemma 3.3. If $A=\left\{a_{x}: x \in \Omega_{A}\right\}$ and $B=\left\{b_{y}: y \in \Omega_{B}\right\}$ are observables, then

$$
\left(\mathcal{L}^{B} \mid \mathcal{L}^{A}\right)^{\wedge}=\left(\left(\mathcal{L}^{B}\right)^{\wedge} \mid\left(\mathcal{L}^{A}\right)^{\wedge}\right)=(B \mid A)
$$

Proof. For all $\rho \in \mathcal{S}(H)$ we obtain

$$
\begin{aligned}
\operatorname{tr}\left[\rho\left(\mathcal{L}^{B} \mid \mathcal{L}^{A}\right)_{y}^{\wedge}\right] & =\operatorname{tr}\left[\left(\mathcal{L}^{B} \mid \mathcal{L}^{A}\right)_{y}(\rho)\right]=\operatorname{tr}\left[\mathcal{L}_{y}^{B}\left(\overline{\mathcal{L}^{A}}(\rho)\right)\right]=\operatorname{tr}\left[\mathcal{L}_{y}^{B}\left(\sum_{x} a_{x}^{1 / 2} \rho a_{x}^{1 / 2}\right)\right] \\
& =\sum_{x} \operatorname{tr}\left[\mathcal{L}_{y}^{B}\left(a_{x}^{1 / 2} \rho a_{x}^{1 / 2}\right)\right]=\sum_{x} \operatorname{tr}\left(b_{y}^{1 / 2} a_{x}^{1 / 2} \rho a_{x}^{1 / 2} b_{y}^{1 / 2}\right) \\
& =\sum_{x} \operatorname{tr}\left(\rho a_{x} \circ b_{y}\right)=\operatorname{tr}\left[\sum_{x}\left(a_{x} \circ b_{y}\right)\right]=\operatorname{tr}\left[\rho(B \mid A)_{y}\right]
\end{aligned}
$$

It follows that $\left(\mathcal{L}^{B} \mid \mathcal{L}^{A}\right)_{y}^{\wedge}=(B \mid A)_{y}$ as hence, the result holds.

Example 9. This example shows that $(\mathcal{J} \mid \mathcal{I})^{\wedge} \neq(\widehat{\mathcal{J}} \mid \widehat{\mathcal{I}})$, in general. Letting $\mathcal{I}_{x}(\rho)=\operatorname{tr}\left(\rho a_{x}\right) \alpha, \mathcal{J}_{y}(\rho)=\operatorname{tr}\left(\rho b_{y}\right) \beta$ be trivial instruments, we obtain

$\operatorname{tr}\left[\rho(\mathcal{J} \mid \mathcal{I})_{y}^{\wedge}\right]=\operatorname{tr}\left[\mathcal{J}_{y}(\overline{\mathcal{I}}(\rho))\right]=\operatorname{tr}\left[\operatorname{tr}\left(\overline{\mathcal{I}}(\rho) b_{y}\right) \beta\right]=\operatorname{tr}\left[\overline{\mathcal{I}}(\rho) b_{y}\right]=\operatorname{tr}\left(\alpha b_{y}\right)$

On the other hand,

$$
\operatorname{tr}\left[\rho(\widehat{\mathcal{J}} \mid \widehat{\mathcal{I}})_{y}\right]=\operatorname{tr}\left[\rho(B \mid A)_{y}\right]=\operatorname{tr}\left[\rho \sum_{x}\left(a_{x} \circ b_{y}\right)\right]
$$

These do not agree, in general. For example, if $\Omega_{\mathcal{I}}=\{x\}$ so that $\mathcal{I}_{x}(\rho)=\alpha$ for all $\rho \in \mathcal{S}(H)$, then we obtain

$$
\operatorname{tr}\left[\rho(\widehat{\mathcal{J}} \mid \widehat{\mathcal{I}})_{y}\right]=\operatorname{tr}\left(\rho b_{y}\right) \neq \operatorname{tr}\left(\alpha b_{y}\right)
$$

in general. 


\section{Mixed Sequential Products and Coexistence}

We have defined sequential products of effects (observables) and of operations (instruments). We now define mixed sequential products for effects with operations and observables with instruments. If $a \in \mathcal{E}(H)$ and $\mathcal{I} \in \mathcal{O}(H)$, we define $a \circ \mathcal{I}=\mathcal{L}^{a} \circ \mathcal{I}$. Thus, $a \circ \mathcal{I} \in \mathcal{O}(H)$ and $(a \circ \mathcal{I})(\rho)=$ $\mathcal{I}\left(a^{1 / 2} \rho a^{1 / 2}\right)$ for all $\rho \in \mathcal{S}(H)$. Notice that $a \circ I=\mathcal{L}^{a}$ and $I \circ \mathcal{I}=\mathcal{I}$. Also, it is easy to check that $a \circ \mathcal{I}$ is additive and affine in the second argument but is not in the first. If $\mathcal{I} \in \mathcal{O}(H)$ has Kraus decomposition $\mathcal{I}(\rho)=\sum B_{i} \rho B_{i}^{*}$ and $a \in \mathcal{E}(H)$, we define $\mathcal{I} \circ a \in \mathcal{E}(H)$ by $\mathcal{I} \circ a=\sum B_{i}^{*} a B_{i}$. This definition does not depend on the particular Kraus operators because if $\mathcal{I}$ also has the decomposition $\mathcal{I}(\rho)=\sum C_{i} \rho C_{i}^{*}$, then for every $\rho \in \mathcal{S}(H)$ we obtain

$$
\begin{aligned}
\operatorname{tr}\left(\rho \sum B_{i}^{*} a B_{i}\right) & =\sum \operatorname{tr}\left(\rho B_{i}^{*} a B_{i}\right)=\sum \operatorname{tr}\left(B_{i} \rho B_{i}^{*} a\right)=\operatorname{tr}\left(\sum B_{i} \rho B_{i}^{*} a\right) \\
& =\operatorname{tr}\left(\sum C_{i} \rho C_{i}^{*} a\right)=\operatorname{tr}\left(\rho \sum C_{i}^{*} a C_{i} i\right)
\end{aligned}
$$

Hence, $\sum B_{i}^{*} a B_{i}=\sum C_{i}^{*} a C_{i}$. It is easy to check that $\mathcal{I} \circ a$ is additive and affine in both variables.

We now extend these definitions to observables and instruments. If $A=$ $\left\{a_{x}: x \in \Omega_{A}\right\}$ is an observable and $\mathcal{I} \in \operatorname{In}(H)$, we define $A \circ \mathcal{I} \in \operatorname{In}(H)$ to have outcome space $\Omega_{A \circ \mathcal{I}}=\Omega_{A} \times \Omega_{\mathcal{I}}$ and

$$
(A \circ \mathcal{I})_{(x, y)}(\rho)=\left(a_{x} \circ \mathcal{I}_{y}\right)(\rho)=\left(\mathcal{L}^{a_{x}} \circ \mathcal{I}_{y}\right)(\rho)=\mathcal{I}_{y}\left(\mathcal{L}^{a_{x}}(\rho)\right)=\mathcal{I}_{y}\left(a_{x}^{1 / 2} \rho a_{x}^{1 / 2}\right)
$$

It is then natural to define $(\mathcal{I} \mid A) \in \operatorname{In}(H)$ to have $\Omega_{(\mathcal{I} \mid A)}=\Omega_{\mathcal{I}}$ and

$$
\begin{aligned}
(\mathcal{I} \mid A)_{y}(\rho) & =\sum_{x}(A \circ \mathcal{I})_{(x, y)}(\rho)=\sum_{x} \mathcal{I}_{y}\left(a_{x}^{1 / 2} \rho a_{x}^{1 / 2}\right)=\mathcal{I}_{y}\left(\sum_{x} a_{x}^{1 / 2} \rho a_{x}^{1 / 2}\right) \\
& =\mathcal{I}_{y}\left(\overline{\mathcal{L}^{A}}(\rho)\right)=\left(\overline{\mathcal{L}^{A}} \circ \mathcal{I}_{y}\right)(\rho)
\end{aligned}
$$

We thus define $(\mathcal{I} \mid A)_{y}=\overline{\mathcal{L}^{A}} \circ \mathcal{I}_{y}$. If $\mathcal{I} \in \operatorname{In}(H)$ has Kraus decomposition $\mathcal{I}_{x}(\rho)=\sum B_{i}^{x} \rho\left(B_{i}^{x}\right)^{*}$ and $A=\left\{a_{x}: x \in \Omega_{A}\right\} \in \mathrm{Ob}(H)$ we define $\mathcal{I} \circ A \in$ $\mathrm{Ob}(H)$ to have outcome space $\Omega_{\mathcal{I} \circ A}=\Omega_{\mathcal{I}} \times \Omega_{A}$ and

$$
(\mathcal{I} \circ A)_{(x, y)}=\mathcal{I}_{x} \circ a_{y}=\sum\left(B_{i}^{x}\right)^{*} a_{y} B_{i}^{x}
$$

It is then natural to define $(A \mid \mathcal{I}) \in \mathrm{Ob}(H)$ to have $\Omega_{(A \mid \mathcal{I})}=\Omega_{A}$ and

$$
(A \mid \mathcal{I})_{y}=\sum_{x}(\mathcal{I} \circ A)_{(x, y)}=\sum_{x, i}\left(B_{i}^{x}\right)^{*} a_{y} B_{i}^{x}=\overline{\mathcal{I}} \circ a_{y}
$$


Theorem 4.1. (i) If $a \in \mathcal{E}(H)$ and $\mathcal{I} \in \mathcal{O}(H)$, then $(a \circ \mathcal{I})^{\wedge}=a \circ \widehat{\mathcal{I}}$. (ii) If $A \in \mathrm{Ob}(H)$ and $\mathcal{I} \in \operatorname{In}(H)$, then $(A \circ \mathcal{I})_{(x, y)}^{\wedge}=a_{x} \circ \widehat{\mathcal{I}}_{y}=(A \circ \widehat{\mathcal{I}})_{(x, y)}$. (iii) If $A \in \mathrm{Ob}(H), \mathcal{I} \in \operatorname{In}(H)$, then $(\mathcal{I} \mid A)^{\wedge}=(\widehat{\mathcal{I}} \mid A)$.

Proof. (i) For all $\rho \in \mathcal{S}(H)$ we have that

$$
\begin{gathered}
\operatorname{tr}\left[\rho(a \circ \mathcal{I})^{\wedge}\right]=\operatorname{tr}[(a \circ \mathcal{I})(\rho)]=\operatorname{tr}\left[\mathcal{I}\left(a^{1 / 2} \rho a^{1 / 2}\right)\right]=\operatorname{tr}\left(a^{1 / 2} \rho a^{1 / 2} \widehat{\mathcal{I}}\right) \\
\operatorname{tr}\left(\rho a^{1 / 2} \widehat{\mathcal{I}} a^{1 / 2}\right)=\operatorname{tr}(\rho a \circ \widehat{\mathcal{I}})
\end{gathered}
$$

and the result follows. (ii) Since $(A \circ \mathcal{I})_{(x, y)}=a_{x} \circ \mathcal{I}_{y}$, by (i) we obtain

$$
(A \circ \mathcal{I})_{(x, y)}^{\wedge}=\left(a_{x} \circ \mathcal{I}_{y}\right)^{\wedge}=a_{x} \circ \widehat{\mathcal{I}}_{y}=(A \circ \widehat{\mathcal{I}})_{(x, y)}
$$

(iii) Applying Theorem 3.1(ii) we have for all $\rho \in \mathcal{S}(H)$ that

$$
\begin{aligned}
\operatorname{tr}\left[\rho(\mathcal{I} \mid A)_{y}^{\wedge}\right] & =\operatorname{tr}\left[(\mathcal{I} \mid A)_{y}(\rho)\right]=\operatorname{tr}\left[\left(\overline{\mathcal{L}^{A}} \circ \mathcal{I}_{y}\right)(\rho)\right]=\left[\rho\left(\mathcal{L}^{A} \circ \mathcal{J}_{y}\right)^{\wedge}\right] \\
& =\operatorname{tr}\left[\rho\left(\sum_{x} \mathcal{L}^{a_{x}} \circ \mathcal{I}_{y}\right)^{\wedge}\right]=\sum_{x} \operatorname{tr}\left[\rho\left(\mathcal{L}^{a_{x}} \circ \mathcal{I}_{y}\right)^{\wedge}\right] \\
& =\sum_{x} \operatorname{tr}\left[\rho\left(a_{x} \circ \widehat{\mathcal{I}}_{y}\right)\right]=\operatorname{tr}\left[\rho \sum_{x}\left(a_{x} \circ \widehat{\mathcal{I}}_{y}\right)\right]=\operatorname{tr}\left[\rho(\widehat{\mathcal{I}} \mid A)_{y}\right]
\end{aligned}
$$

We conclude that $(\mathcal{I} \mid A)^{\wedge}=(\widehat{\mathcal{I}} \mid A)$.

We have seen that if $A, B \in \mathrm{Ob}(H)$, then $A \circ \mathcal{L}^{B}=\mathcal{L}^{A} \circ \mathcal{L}^{B}$. On the other hand

$$
\left(\mathcal{L}^{A} \circ B\right)_{(x, y)}=\mathcal{L}_{x}^{A} \circ b_{y}=a_{x}^{1 / 2} b_{y} a_{x}^{1 / 2}=a_{x} \circ b_{y}=(A \circ B)_{(x, y)}
$$

Hence, $\mathcal{L}^{A} \circ B=A \circ B$. We now treat trivial and semi-trivial instruments.

Theorem 4.2. (i) Let $a \in \mathcal{E}(H)$ and let $\mathcal{I}$ be a trivial operation $\mathcal{I}(\rho)=$ $\operatorname{tr}(\rho b) \alpha$. Then $\mathcal{I} \circ A=\operatorname{tr}(\alpha a) b$ and $a \circ \mathcal{I}$ is trivial with state $\alpha$ and effect $a \circ b$. (ii) Let $A=\left\{a_{x}: x \in \Omega_{A}\right\}$ be an observable and let $\mathcal{I}$ be semi-trivial with states $\alpha_{y}$ and observable $B$. Then $(\mathcal{I} \circ A)_{(x, y)}=\operatorname{tr}\left(\alpha_{x} a_{y}\right) b_{x}$ and $A \circ \mathcal{I}$ is semi-trivial with states $\alpha_{y}$ and observable $A \circ B$. (iii) Let $A=\left\{a_{x}: x \in \Omega_{A}\right\}$ be an observable and let $\mathcal{I}$ be trivial with state $\alpha$ and observable $B$. Then $(\mathcal{I} \circ a)_{(x, y)}=\operatorname{tr}\left(\alpha a_{y}\right) b_{x}$ and $A \circ \mathcal{I}$ is trivial with state $\alpha$ and observable $A \circ B$. 
Proof. (i) Letting $\mathcal{I}$ have Kraus decomposition $\mathcal{I}(\rho)=\sum A_{i} \rho A_{i}^{*}$ with $\sum A_{i}^{*} A_{i} \leq$ $I$ we have for all $\rho \in \mathcal{S}(H)$ that

$$
\begin{aligned}
\operatorname{tr}(\rho \mathcal{I} \circ a) & =\operatorname{tr}\left(\rho \sum A_{i}^{*} a A_{i}\right)=\sum \operatorname{tr}\left(\rho A_{i}^{*} a A_{i}\right)=\sum \operatorname{tr}\left(A_{i} \rho A_{i}^{*} a\right) \\
& =\operatorname{tr}\left(\sum A_{i} \rho A_{i}^{*} a\right)=\operatorname{tr}[\mathcal{I}(\rho) a]=\operatorname{tr}[\operatorname{tr}(\rho b) \alpha a] \\
& =\operatorname{tr}(\rho b) \operatorname{tr}(\alpha a)=\operatorname{tr}[\rho \operatorname{tr}(\alpha a) b]
\end{aligned}
$$

Hence, $\mathcal{I} \circ a=\operatorname{tr}(\alpha a) b$. Moreover, for all $\rho \in \mathcal{S}(H)$ we obtain

$$
\begin{aligned}
(a \circ \mathcal{I})(\rho) & =\mathcal{I}\left(a^{1 / 2} \rho a^{1 / 2}\right)=\operatorname{tr}\left(a^{1 / 2} \rho a^{1 / 2} b\right) \alpha=\operatorname{tr}\left(\rho a^{1 / 2} b a^{1 / 2}\right) \alpha \\
& =\operatorname{tr}(\rho a \circ b) \alpha
\end{aligned}
$$

Thus, $a \circ \mathcal{I}$ is trivial with state $\alpha$ and effect $a \circ b$.

(ii) Letting $\mathcal{I}_{x}$ have Kraus decomposition $\mathcal{I}_{x}(\rho)=\sum A_{i}^{x} \rho\left(A_{i}^{x}\right)^{*}$, we have from (i) that

$$
\operatorname{tr}\left[\rho(\mathcal{I} \circ A)_{(x, y)}\right]=\operatorname{tr}\left(\rho \mathcal{I}_{x} \circ a_{y}\right)=\operatorname{tr}\left[\operatorname{tr}\left(\alpha_{x} a_{y}\right) b_{x}\right]
$$

Therefore, $(\mathcal{I} \circ A)_{(x, y)}=\operatorname{tr}\left(\alpha_{x} a_{y}\right) b_{x}$. Moreover, by (i) we have for all $\rho \in$ $\mathcal{S}(H)$ that

$$
(A \circ \mathcal{I})_{(x, y)}(\rho)=\left(a_{x} \circ \mathcal{I}_{y}\right)(\rho)=\operatorname{tr}\left(\rho a_{x} \circ b_{y}\right) \alpha_{y}
$$

Hence, $A \circ \mathcal{I}$ is semi-trivial with states $\alpha_{y}$ and observable $(A \circ B)_{(x, y)}=a_{x} \circ b_{y}$. (iii) follows from (ii).

Let $\mathcal{I} \in \mathcal{O}(H)$ be a Kraus with $\mathcal{I}(\rho)=S \rho S^{*}$ and let $a \in \mathcal{E}(H)$. Then $\mathcal{I} \circ a=S^{*} a S$ and

$$
(a \circ \mathcal{I})(\rho)=\mathcal{I}\left(a^{1 / 2} \rho a^{1 / 2}\right)=S a^{1 / 2} \rho a^{1 / 2} S^{*}
$$

Thus, $a \circ \mathcal{I} \in \mathcal{O}(H)$ is Kraus with Kraus operator $S a^{1 / 2}$. More generally, if $\mathcal{I} \in \operatorname{In}(H)$ is Kraus with $\mathcal{I}_{x}(\rho)=S_{x} \rho S_{x}^{*}$ and $A=\left\{a_{y}: y \in \Omega_{A}\right\} \in \mathrm{Ob}(H)$, then

$$
(\mathcal{I} \circ A)_{(x, y)}=\mathcal{I}_{x} \circ a_{y}=S_{x}^{*} a_{y} S_{x}
$$

and

$$
(A \circ \mathcal{I})_{(x, y)}(\rho)=\left(a_{y} \circ \mathcal{I}_{x}\right)(\rho)=\mathcal{I}_{x}\left(a_{y}^{1 / 2} \rho a_{y}^{1 / 2}\right)=S_{x} a_{y}^{1 / 2} \rho a_{y}^{1 / 2} S_{x}^{*}
$$

Thus, $A \circ \mathcal{I} \in \operatorname{In}(H)$ is Kraus with Kraus operators $S_{x} a_{y}^{1 / 2}$. 
Example 10. If $\mathcal{I} \in \mathcal{O}(H)$ is a channel, it is easy to check that

$J(a)=\mathcal{I} \circ a$ is a convex, effect algebra morphism on $\mathcal{E}(H)$. We now show that $J$ need not be a monomorphism. That is, if $J(a) \perp J(b)$, then we need not have $a \perp b$. Let $\left\{\psi_{1}, \psi_{2}\right\}$ be an orthonormal basis for $\mathbb{C}^{2}$ and let $a_{1}=\left|\psi_{1}\right\rangle\left\langle\psi_{1}\left|, a_{2}=\right| \psi_{2}\right\rangle\left\langle\psi_{2}\right|$. Then $\mathcal{I}(\rho)=a_{1} \rho a_{1}+a_{2} \rho a_{2}$ is a channel. Letting $d=\left|\psi_{1}+\psi_{2}\right\rangle\left\langle\psi_{1}+\psi_{2}\right|$ we have that

$$
d=2\left|\frac{\psi_{1}+\psi_{2}}{\sqrt{2}}\right\rangle\left\langle\frac{\psi_{1}+\psi_{2}}{\sqrt{2}}\right|
$$

Hence, $d$ is twice a one-dimensional projection so $d \not \leq I$. Letting $a=b=\frac{1}{2} d$ we have that $a, b \in \mathcal{E}(H)$ and $a+b=d \not \leq I$ so $a \not \perp b$. However,

$$
\begin{aligned}
J(a)+J(b)= & J(d) \\
= & \left|\psi_{1}\right\rangle\left\langle\psi_{1}|| \psi_{1}+\psi_{2}\right\rangle\left\langle\psi_{1}+\psi_{2}|| \psi_{1}\right\rangle\left\langle\psi_{1}\right| \\
& \quad+\left|\psi_{2}\right\rangle\left\langle\psi_{2}|| \psi_{1}+\psi_{2}\right\rangle\left\langle\psi_{1}+\psi_{2}|| \psi_{2}\right\rangle\left\langle\psi_{2}\right| \\
= & \left|\psi_{1}\right\rangle\left\langle\psi_{1}|+| \psi_{2}\right\rangle\left\langle\psi_{2}\right|=I
\end{aligned}
$$

so $J(a) \perp J(b)$.

An observable $B=\left\{b_{y}: y \in \Omega_{B}\right\}$ is part [2] of an observable $A=$ $\left\{a_{x}: x \in \Omega_{A}\right\}$ if there exists a surjection $f: \Omega_{A} \rightarrow \Omega_{B}$ such that

$$
b_{y}=A_{f^{-1}(y)}=\sum\left\{a_{x}: f(x)=y\right\}
$$

We then write $B=f(A)$. Two observables $B, C$ coexist [1, 8, 9] if there exists an observable $A$ such that $B=f(A), C=g(A)$. Thus, $B$ and $C$ coexist if they can be measured by applying a single observable $A$. If $A, B \in \mathrm{Ob}(H)$ with $A=\left\{a_{x}: x \in \Omega_{A}\right\}, B=\left\{b_{y}: y \in \Omega_{B}\right\}$, define $f: \Omega_{A} \times \Omega_{B} \rightarrow \Omega_{B}$ by $f(x, y)=y$. Then

$$
(B \mid A)_{y}=\sum_{x} a_{x} \circ b_{y}=\sum_{x}(A \circ B)_{(x, y)}=\sum\left\{(A \circ B)_{(x, y)}: f(x, y)=y\right\}
$$

Therefore, $(B \mid A)_{y}=(A \circ B)_{f^{-1}(y)}$ so $(B \mid A)=f(A \circ B)$. We conclude that $(B \mid A)$ and $A \circ B$ coexist. Also, $A$ and $A \circ B$ coexist because $a_{x}=\sum_{y} a_{x} \circ b_{y}$. Thus, $(B \mid A)$ and $A$ coexist.

If $\mathcal{I}, \mathcal{J} \in \operatorname{In}(H)$, we have the instrument $\mathcal{I} \circ \mathcal{J}$ given by $(\mathcal{I} \circ \mathcal{J})_{(x, y)}(\rho)=$ $\mathcal{J}_{y}\left(\mathcal{I}_{x}(\rho)\right)$ with channel $\overline{\mathcal{I} \circ \mathcal{J}}=\overline{\mathcal{I}} \circ \overline{\mathcal{J}}$ and instrument $(\mathcal{J} \mid \mathcal{I})$ given by 
$(\mathcal{J} \mid \mathcal{I})_{y}(\rho)=\mathcal{J}_{y}(\overline{\mathcal{I}}(\rho))$ with channel $\overline{(\mathcal{J} \mid \mathcal{I})}=\overline{\mathcal{I}} \circ \overline{\mathcal{J}}$. We say that $\mathcal{J}$ is part of $\mathcal{I}$ if there exists a surjection $f: \Omega_{\mathcal{I}} \rightarrow \Omega_{\mathcal{J}}$ such that

$$
\mathcal{J}_{y}=\mathcal{I}_{f^{-1}(y)}=\sum\left\{\mathcal{I}_{x}: f(x)=y\right\}
$$

We then write $\mathcal{J}=f(\mathcal{I})$. As with observables, we say that $\mathcal{J}, \mathcal{K} \in \operatorname{In}(H)$ coexist if there exists an instrument $\mathcal{I}$ such that $\mathcal{J}=f(\mathcal{I}), \mathcal{K}=g(\mathcal{I})$. Moreover, we have that $(\mathcal{J} \mid \mathcal{I})$ and $\mathcal{I} \circ \mathcal{J}$ coexist. However, $\mathcal{I}_{x} \neq \sum_{y} \mathcal{I}_{x} \circ \mathcal{J}_{y}$ in general, so $(\mathcal{J} \mid \mathcal{I})$ and $\mathcal{I}$ may not coexist.

Lemma 4.3. (i) $f(\mathcal{I})^{\wedge}=f(\widehat{\mathcal{I}})$. (ii) If $\mathcal{J}, \mathcal{K}$ coexist, the $\widehat{\mathcal{J}}, \widehat{\mathcal{K}}$ coexist.

Proof. (i) For all $\rho \in \mathcal{S}(H)$ we have that

$$
\begin{aligned}
\operatorname{tr}\left[\rho f\left(\mathcal{I}_{y}^{\wedge}\right]\right. & =\operatorname{tr}\left[f(\mathcal{I})_{y}(\rho)\right]=\operatorname{tr}\left[\mathcal{I}_{f^{-1}(y)}(\rho)\right]=\operatorname{tr}\left[\sum_{f(x)=y} \mathcal{I}_{x}(\rho)\right] \\
& =\sum_{f(x)=y} \operatorname{tr}\left[\mathcal{I}_{x}(\rho)\right]=\sum_{f(x)=y} \operatorname{tr}\left(\rho \widehat{\mathcal{I}}_{x}\right)=\operatorname{tr}\left(\rho \sum_{f(x)=y} \widehat{\mathcal{I}}_{x}\right)=\operatorname{tr}\left[\rho f(\widehat{\mathcal{I}})_{y}\right]
\end{aligned}
$$

Hence, $f(\mathcal{I})^{\wedge}=f(\widehat{\mathcal{I}})$. (ii) If $\mathcal{J}, \mathcal{K}$ coexist, the $\mathcal{J}=f(\mathcal{I}), \mathcal{K}=g(\mathcal{I})$ for some $\mathcal{I} \in \operatorname{In}(H)$. By (i) we obtain $\widehat{\mathcal{J}}=f(\widehat{\mathcal{I}})$ and $\widehat{\mathcal{K}}=g(\widehat{\mathcal{I}})$ so $\widehat{\mathcal{J}}$ and $\widehat{\mathcal{K}}$ coexist.

Lemma 4.4. (i) If $\mathcal{I}_{x}(\rho)=\operatorname{tr}\left(\rho a_{x}\right) \alpha$ is trivial and $\mathcal{J}=f(\mathcal{I})$, then $\mathcal{J}$ is trivial with state $\alpha$ and observable $f(A)$. (ii) If $\mathcal{J}, \mathcal{K}$ are trivial with the same state $\alpha$ and $\widehat{\mathcal{J}}, \widehat{\mathcal{K}}$ coexist, then $\mathcal{J}, \mathcal{K}$ coexist.

Proof. (i) For all $\rho \in \mathcal{S}(H)$ we obtain

$\mathcal{J}_{y}(\rho)=\sum_{f(x)=y} \mathcal{I}_{x}(\rho)=\sum_{f(x)=y} \operatorname{tr}\left(\rho a_{x}\right) \alpha=\operatorname{tr}\left(\rho \sum_{f(x)=y} a_{x}\right) \alpha=\operatorname{tr}\left[\rho f(A)_{y}\right] \alpha$

Hence, $\mathcal{J}$ is trivial with state $\alpha$ and observable $f(A)$. (ii) Let $\mathcal{J}_{y}(\rho)=$ $\operatorname{tr}\left(\rho b_{y}\right) \alpha, \mathcal{K}_{z}(\rho)=\operatorname{tr}\left(\rho c_{z}\right) \alpha$ be trivial with the same state $\alpha$. Since $\mathcal{J}, \mathcal{K}$ coexist there exists an observable $A=\left\{a_{x}: x \in \Omega_{A}\right\}$ such that $\widehat{\mathcal{J}}=f(A)$, $\widehat{\mathcal{K}}=g(A)$. Letting $\mathcal{I} \in \operatorname{In}(H)$ be defined by $\mathcal{I}_{x}(\rho)=\operatorname{tr}\left(\rho a_{x}\right) \alpha$ we obtain from (i) that

$$
\mathcal{J}_{y}=\operatorname{tr}\left(\rho \widehat{\mathcal{J}}_{y}\right) \alpha=\operatorname{tr}\left[\rho f(A)_{y}\right] \alpha=f(\mathcal{I})_{y}(\rho)
$$


and

$$
\mathcal{K}_{z}(\rho)=\operatorname{tr}\left(\rho \widehat{\mathcal{K}}_{z}\right) \alpha=\operatorname{tr}\left[\rho g(A)_{z}\right] \alpha=g(\mathcal{I})_{z}(\rho)
$$

Hence, $\mathcal{J}=f(\mathcal{I}), \mathcal{K}=g(\mathcal{I})$ so $\mathcal{J}, \mathcal{K}$ coexist

\section{References}

[1] P. Busch, M. Grabowski and P. Lahti, Operational Quantum Physics, Springer-Verlag, Berlin, 1995.

[2] S. Fillipov, T. Heinosaari and L. Leppäjärvi, Simulability of observables in general probabilistic theories, Phys. Rev.A97, 062102 (2018).

[3] S. Gudder and R. Greechie, Sequential Products on effect algebras, Rep. Math. Phys. 49, 87-111 (2002).

[4] S. Gudder and G. Nagy, Sequential quantum measurements, J. Math. Phys. 42, 5212-5222 (2001).

[5] S. Gudder, Quantum instruments and conditioned observables, arXiv:quant-ph 2005.08117 (2020).

[6] — , Parts and composites of quantum systems, arXiv:quant-ph 2009.07371 (2020).

[7] — Combinations of quantum observables and instruments, arXiv:quant-ph 2010.08025 (2020)

[8] T. Heinosaari and M. Ziman, The Mathematical Language of Quantum Theory, Cambridge University Press, Cambridge, 2012.

[9] T. Heinosaari, D. Reitzner, R. Stano and M. Ziman, Coexistence of quantum operations, J. Phys. A42, 365302 (2009).

[10] K. Kraus, States, Effects and Operations, Springer-Verlag, Berlin, 1983.

[11] P. Lahti, Coexistence and joint measurability in quantum mechanics, Int. J. Theor. Phys. 42, 893-906 (2003). 
[12] G. Lüders, Über due Zustandsänderung durch den Messprozess, Ann. Physik 6, 322-328 (1951).

[13] M. Nielson and I. Chuang, Quantum Computation and Quantum Information, Cambridge University Press, Cambridge, 2000. 